\title{
ILLINOIS.
}

\section{PRIMARY TRAVERSE.}

Beardstown, Belvidére, Carbondale, Chandleville, Dixon, Dongola, Genoa, Harvard, Havana, Jonesboro, Kings, Kirkland, Mound City, Oregon, Rockford, Thebes, and Vermont quadrangles.

ALEXANDER, BOONE, OQLE, PULASKI, SCHUYLER, UNION, AND WINNEBAGO COUNTIES.

The primary traverse was done by J. H. Wilson in 1916 and F. W. Crisp in 1917, as indicated at the beginning of each quadrangle list.

For other positions in Illinois see United States Geological Survey Bulletins 201, 216, 245, 276, 310, 440, 496, 551, and 644, Appendix to the Eighteenth and Nineteenth Annual Reports of the Director, United States Coast and Geodetic Survey, Special Publication 30, Appendix EEE, Report of the Chief of Engineers for 1902, Mississippi River Commission Report for 1883, 1888, 1892, 1893, and 1894, and Appendix 22, House Document 50, Sixty-first Congress.

\section{BEARDSTOWN QUADRANGLE.}

Along highways near north border of quadrangle. (By J. H. Wilson in 1916.)

\begin{tabular}{|c|c|c|c|}
\hline Station. & Latitude. & Longitude. & $\begin{array}{l}\text { Distance } \\
\text { between } \\
\text { stations. }\end{array}$ \\
\hline 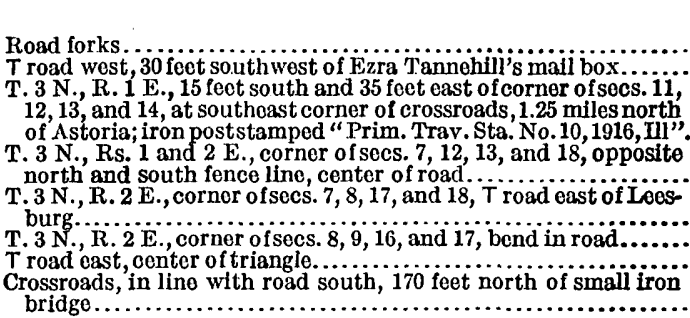 & $\begin{array}{r}01 " \text { ' } \\
401444.8 \\
\because 401441.5 \\
401448.6 \\
401446.9 \\
401446.3 \\
401447.0 \\
401433.4 \\
401427.8\end{array}$ & $\begin{array}{lll}\circ & \prime \prime \prime \\
90 & 24 & 45.1 \\
90 & 22 & 14.8 \\
90 & 21 & 18.8 \\
90 & 20 & 10.3 \\
90 & 19 & 03.0 \\
90 & 17 & 48.8 \\
90 & 16 & 57.7 \\
90 & 15 & 44.3\end{array}$ & $\begin{array}{r}\text { Fcet. } \\
\ldots \ldots \ldots \\
4,402 \\
5,319\end{array}$ \\
\hline
\end{tabular}

Magnetic declination for north border of quadrangle, $5^{\circ} 44^{\prime} \mathrm{E}$.

BELVIDERE QUADRANGIE.

Along highways near south border of quadrangle. (By F. W. Crisp in 1917.)

\begin{tabular}{|c|c|c|c|}
\hline Station. & $\begin{array}{l}\text { Latitude. } \\
\text { s. }\end{array}$ & Longitude. & $\begin{array}{l}\text { Distance } \\
\text { between } \\
\text { stations. }\end{array}$ \\
\hline & 0,11 & - , " & Fect. \\
\hline 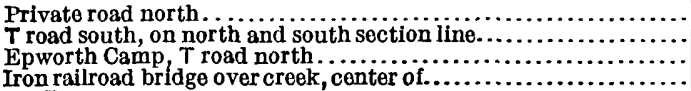 & $\begin{array}{lll}42 & 15 & 15.1 \\
42 & 15 & 10.4 \\
42 & 15 & 08.3 \\
42 & 15 & 16.3\end{array}$ & $\begin{array}{lll}88 & 45 & 09.7 \\
88.45 & 50.1 \\
88 & 46 & 07.4 \\
88 & 46 & 14.5\end{array}$ & $\begin{array}{r}3,080 \\
1,320 \\
961\end{array}$ \\
\hline $\begin{array}{l}\text { Do } \\
\text { Belvidere, } 1.5 \text { miles east of, railiroad crossing } \ldots \ldots \ldots \ldots \ldots \ldots \ldots \ldots\end{array}$ & $\begin{array}{lll}42 & 15 & 16.1 \\
42 & 15 & 04.9\end{array}$ & $\begin{array}{lll}88 & 47 & 39.2 \\
88 & 48 & 54.7\end{array}$ & $\begin{array}{l}6,370 \\
5,798\end{array}$ \\
\hline 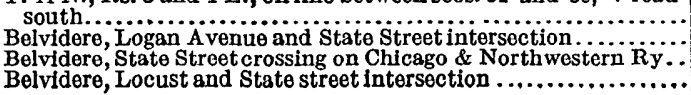 & $\begin{array}{lll}42 & 15 & 09.4 \\
42 & 15 & 19.1 \\
42 & 15 & 24.2 \\
42 & 15 & 26.8\end{array}$ & $\begin{array}{lll}88 & 49 & 18.5 \\
88 & 50 & 19.5 \\
88 & 50 & 24.5 \\
88 & 50 & 27.0\end{array}$ & $\begin{array}{r}1,840 \\
4,694 \\
640 \\
320\end{array}$ \\
\hline
\end{tabular}


BELVIDERE QUADRANGLE-Continued.

Along highways near south border of quadrangle-Continued.

\begin{tabular}{|c|c|c|c|}
\hline Station. & Latitude. & Longitude. & $\begin{array}{l}\text { Distance } \\
\text { between } \\
\text { stations. }\end{array}$ \\
\hline 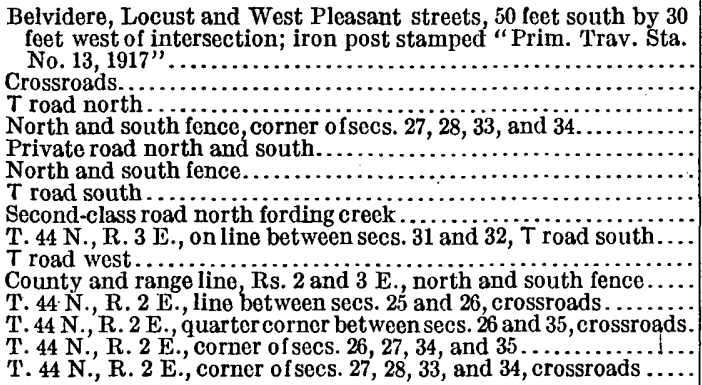 & $\begin{array}{ccc}\circ & \prime & \prime \prime \\
42 & 15 & 16.3 \\
42 & 15 & 13.0 \\
42 & 15 & 18.1 \\
42 & 15 & 17.5 \\
42 & 15 & 17.1 \\
42 & 15 & 17.0 \\
42 & 15 & 15.3 \\
42 & 15 & 05.5 \\
42 & 15 & 07.9 \\
42 & 15 & 20.9 \\
42 & 15 & 15.5 \\
42 & 15 & 14.5 \\
42 & 15 & 13.6 \\
42 & 15 & 13.0 \\
42 & 15 & 12.0\end{array}$ & $\begin{array}{ccc}\circ & \prime & \prime \prime \\
88 & 50 & 44.9 \\
88 & 51 & 39.3 \\
88 & 51 & 51.6 \\
88 & 52 & 49.6 \\
88 & 53 & 25.0 \\
88 & 53 & 42.7 \\
88 & 54 & 02.1 \\
88 & 54 & 34.3 \\
88 & 55 & 11.3 \\
88 & 55 & 45.1 \\
88 & 56 & 26.1 \\
88 & 57 & 18.7 \\
88 & 58 & 11.9 \\
88 & 58 & 47.2 \\
88 & 59 & 57.8\end{array}$ & $\begin{array}{r}\text { Feet. } \\
1,715 \\
4,106 \\
1,065 \\
4,360 \\
2,660 \\
1,330 \\
1,473 \\
2,615 \\
2,800 \\
2,859 \\
3,133 \\
3,959 \\
4,000 \\
2,660 \\
5,305\end{array}$ \\
\hline
\end{tabular}

Magnetic declination along south border of quadrangle, $2.5^{\circ} \mathrm{E}$.

Along east border of quadrangle. (By F. W. Crisp in 1917.)

T. 44 N., R. 4 E., corner of secs. $3,4,9$, and 10 , crossroads........
Tps. 44 and 45 N., R. 4 E., corner of secs. $3,4,33$, and 34 , crossroads.. Tps. 44 and 45 N., R. 4 E., corner of secs. 3,4 , 33, and 34 , crossroads.
T. 45 N., R. 4 F., quarter corner between sees. 33 and 34 , T road 1. $45 \mathrm{Nest}$

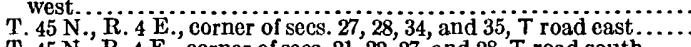
T. 45 N., R. 4 E., corner of secs. $21,22,27$, and 28 , T road south..... T. 45 N., R. 4 E., quarter corner between secs. 22 and 27 .

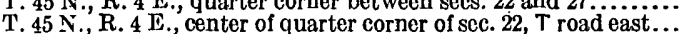

T. 45 N., R. 4 E., quarter corner between sees. 15 and $22, T$ road T. 45 N., $\mathbf{R} .4$ E., in sec. 15,20 feet north by 15 feet east of crossT'. 45 N., $\mathbf{R} .4$ E., in sec. 15,20 feet north by 15 feet east of cross-
roads; iron post stamped "Prim. Trav. Sta. No. 14, 1917"......

\begin{tabular}{|c|c|c|}
\hline $\begin{array}{lll}42 & 18 & 47.8 \\
42 & 19 & 41.0\end{array}$ & $\begin{array}{lll}88 & 45 & 50.6 \\
88 & 45 & 50.4\end{array}$ & 5,390 \\
\hline $\begin{array}{lll}42 & 20 & 07.0 \\
42 & 20 & 33.5 \\
42 & 21 & 25.5 \\
42 & 21 & 25.6 \\
42 & 21 & 51.5\end{array}$ & $\begin{array}{lll}88 & 45 & 50.7 \\
88 & 45 & 51.0 \\
88 & 45 & 51.4 \\
88 & 45 & 15.7 \\
88 & 45 & 16.0\end{array}$ & $\begin{array}{l}2,630 \\
2,690 \\
5,259 \\
2,680 \\
2,620\end{array}$ \\
\hline $\begin{array}{lll}42 & 22 & 17.5\end{array}$ & $\begin{array}{lll}88 & 45 & 15.7\end{array}$ & 2,640 \\
\hline 422224.9 & $\begin{array}{llll}88 & 45 & 15.7\end{array}$ & 7 \\
\hline
\end{tabular}

West along highways through center of quadrangle. (By F. W. Crisp in 1917.)

Prim. Trav. Sta. No. 4 (previously described)

T. 45 N., R. 4 E., quarter corner between secs. 20 and $21 . . . \ldots \ldots . . . .$.

Crossing of north and south road and Chicago \&. Northwestern $\mathrm{Ry}$. T. $45 \mathrm{~N}$., R. 4 E., quarter corner between secs. 17 and 20, crossroads.

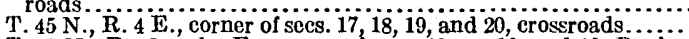
T. 45 N., Rs. 3 and 4 E., corner of secs. 13, 24, 18, and 19, Poplar

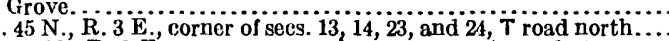
T. 45 N., R. 3 E., corner of secs. 13 , 14 , 23 , and 24 , T road north.... roads.

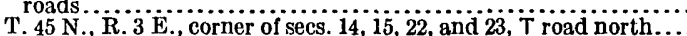
T. 45 N., R. 3 E., quarter corner between secs. 15 and $22 . . . . . . .$.

$T$ road to north.

Caledonia, 345 feet northeast of railroad station, nt north west corner of old store, on cast side street, at sharp angle to east; iron post stamped "Prim. Trav. Sta. No. 15, 1917"................

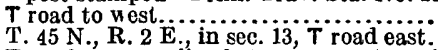

$T$ road west, on line between secse 13 and 24

T. 45 N., R. 2 E. corner of secs. 13, 14, 23 , and 24 .

Dim $T$ road north .................................

T. 45 N., R. 2 F., quarter corner between secs. 14 and $23, T$ road T. 45 N., R. 2 E., corner of secs. $14,15,22, \ldots \ldots \ldots \ldots$ and 23 , private road T. $45 \mathrm{~N} ., \mathrm{R} .2 \mathrm{E}$. , corner of secs. 14, 15,22 , and 23 , private road

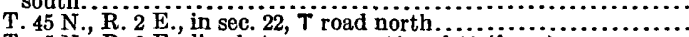

T. 45 N., R. 2 E., line between secs. 21 and 22 (fence).

\begin{tabular}{|c|c|c|}
\hline $\begin{array}{lll}42 & 22 & 24.9 \\
42 & 21 & 51.2 \\
42 & 22 & 01.2\end{array}$ & $\begin{array}{lll}88 & 45 & 16.7 \\
88 & 47 & 01.5 \\
88 & 47 & 36.3\end{array}$ & $\begin{array}{l}\dddot{8}, 649 \\
2,797\end{array}$ \\
\hline $\begin{array}{lll}42 & 22 & 17.4 \\
42 & 22 & 17.4\end{array}$ & $\begin{array}{lll}88 & 47 & 36.2 \\
88 & 48 & 11.0\end{array}$ & $\begin{array}{l}1,640 \\
2,610\end{array}$ \\
\hline $\begin{array}{lll}42 & 22 & 17.9 \\
42 & 22 & 17.2\end{array}$ & $\begin{array}{lll}88 & 49 & 18.9 \\
88 & 50 & 29.5\end{array}$ & $\begin{array}{l}5,100 \\
5,300\end{array}$ \\
\hline $\begin{array}{lll}42 & 22 & 16.9 \\
42 & 22 & 16.5 \\
42 & 22 & 16.2 \\
42 & 22 & 16.0\end{array}$ & $\begin{array}{lll}88 & 51 & 04.6 \\
88 & 51 & 39.9 \\
88 & 52 & 15.1 \\
88 & 52 & 32.8\end{array}$ & $\begin{array}{l}2,640 \\
2,650 \\
2,640 \\
1,330\end{array}$ \\
\hline $\begin{array}{lll}42 & 22 & 15.2 \\
42 & 22 & 41.0 \\
42 & 22 & 39.6 \\
42 & 22 & 13.5 \\
42 & 22 & 13.0 \\
42 & 22 & 12.9\end{array}$ & $\begin{array}{lll}88 & 53 & 29.2 \\
88 & 54 & 28.1 \\
88 & 56 & 52.3 \\
88 & 56 & 43.9 \\
88 & 57 & 35.8 \\
88 & 57 & 57.5\end{array}$ & $\begin{array}{r}4,234 \\
5,140 \\
10,820 \\
2,720 \\
3,900 \\
1,630\end{array}$ \\
\hline $\begin{array}{lll}42 & 22 & 12.8\end{array}$ & $8858 \quad 11.1$ & 1,020 \\
\hline $\begin{array}{lll}42 & 22 & 12.6 \\
42 & 22 & 00.9 \\
42 & 22 & 58.5\end{array}$ & $\begin{array}{lll}88 & 58 & 46.3 \\
88 & 59 & 39.6 \\
88 & 59.57 .3\end{array}$ & $\begin{array}{r}2,650 \\
4,170 \\
1,356\end{array}$ \\
\hline
\end{tabular}

Magnetic declination in center of quadrangle, $3.2^{\circ} \mathrm{E}$. 
CaRBONDALE QUADRANGLE.

NoTE. - An adjustment of $3^{\prime \prime}$ in latitude and $0.5^{\prime \prime}$ in longitude has been applied between the east and west boarders of this quadrangle.

East along hlghways near south border of quadrangle. (By J. H. Wilson In 1916.)

\begin{tabular}{|c|c|c|c|}
\hline Station. & Latitude. & Longitude. & $\begin{array}{l}\text { Distance } \\
\text { between } \\
\text { stations. }\end{array}$ \\
\hline 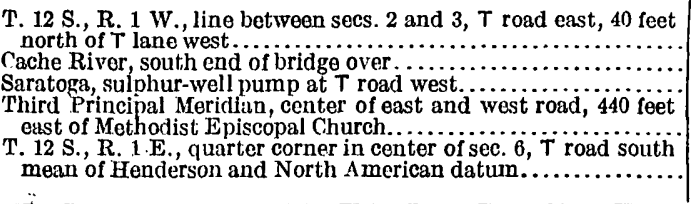 & $\begin{array}{lcc}\circ & \prime & \prime \prime \\
37 & 30 & 07.8 \\
37 & 30 & 16.5 \\
37 & 30 & 35.1 \\
37 & 30 & 25.3 \\
37 & 30 & 19.2\end{array}$ & $\begin{array}{lcc}\circ & \prime \prime \\
89 & 11 & 33.9 \\
89 & 10 & 15.1 \\
89 & 09 & 23.0 \\
89 & 00 & 17.1 \\
89 & 08 & 41.5\end{array}$ & 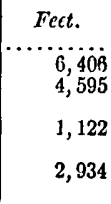 \\
\hline
\end{tabular}

Magnetic declination near south border of quadrangle, $5^{\circ} \mathrm{E}$.

\section{CHANDLEVILLE QUADRANGLE.}

Along highways near northwest corner of quadrangle. (By J. H. Wilson in 1916.)

\begin{tabular}{|c|c|c|c|}
\hline Station. & Latitude. & Longitude. & $\begin{array}{l}\text { Distance } \\
\text { botween } \\
\text { stations. }\end{array}$ \\
\hline $\begin{array}{l}\text { T. } 3 \text { N., R. } 2 \text { E., in north half of sec. } 13 \text {, at road forks, at south edge } \\
\text { of road, } 15 \text { feet east of James Walraven's mail hox, } 35 \text { feet south- } \\
\text { west of walnut tree, } 4,200 \text { feet south of small iron bridge over } \\
\text { creek; iron post stamped "Prim. Trav. Sta. No. 11, 1916, Ill.".. }\end{array}$ & $\begin{array}{ccc}\circ & 1 & \prime \prime \\
40 & 14 & 31.2\end{array}$ & 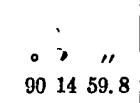 & $\begin{array}{c}\text { Fect. } \\
\ldots \ldots \ldots \ldots\end{array}$ \\
\hline
\end{tabular}

Magnetic declination for northwest corner of quadrangle, $5^{\circ} 44^{\prime} \mathrm{E}$.

DIXON QUADRANGLE.

Near northeast. corner of quadrangle. (By F. W. Crisp in 1917.)

\begin{tabular}{|c|c|c|c|}
\hline Station. & Latitude. & Longitude. & $\begin{array}{l}\text { Distance } \\
\text { between } \\
\text { stations. }\end{array}$ \\
\hline 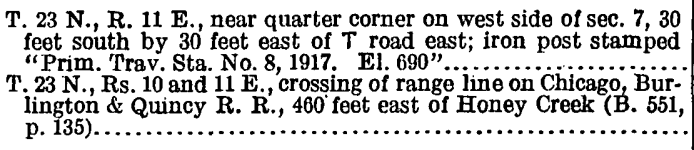 & $\begin{array}{ccc}\circ & \prime & \prime \prime \\
41 & 59 & 40.1 \\
41 & 59 & 28.8\end{array}$ & $\begin{array}{ccc}\circ & \prime & \prime \prime \\
89 & 16 & 45.7 \\
89 & 16 & 46.6\end{array}$ & $\begin{array}{l}\text { Fect. } \\
\quad 1,145\end{array}$ \\
\hline
\end{tabular}

Magnetic declination for northeast corner of quadrangle, $4.5^{\circ} \mathrm{E}$. (one observation).

\section{DoNGOLA QUAdRANGIE.}

Nore.-An adjustment of approximately $3^{\prime \prime}$ in latitude and $1^{\prime \prime}$ in longitude has been applled between the east and west borders of this quadrangle because of the difference in Henderson and North American datum in which respectively positions on the east and west borders of the quadrangle are hased. The correction to Henderson datum is + in latitude and - in longitude.

East along highways near north border of quadrangle. (By J. H. Wilson in 1916.)

\begin{tabular}{|c|c|c|c|}
\hline Station. & Latitude. & Longitude. & $\begin{array}{l}\text { Distance } \\
\text { between } \\
\text { stations. }\end{array}$ \\
\hline 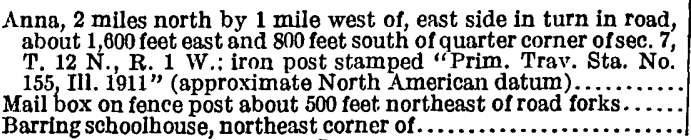 & $\begin{array}{ccc}\circ & \prime & \prime \prime \\
37 & 29 & 20.0 \\
37 & 29 & 19.5 \\
37 & 29 & 47.1\end{array}$ & $\begin{array}{ccc}\circ & \prime \prime \\
89 & 15 & 50.7 \\
89 & 13 & 43.1 \\
89 & 12 & 19.7\end{array}$ & $\begin{array}{c}\text { Feet. } \\
\cdots \\
10,278 \\
7,271\end{array}$ \\
\hline
\end{tabular}


DONGOLA QUADRANGLE-Continued.

East along highways near north border of quadrangle-Continued.

\begin{tabular}{|c|c|c|c|}
\hline Station. & Latitude. & Longitude. & $\begin{array}{l}\text { Distance } \\
\text { between } \\
\text { stations. }\end{array}$ \\
\hline 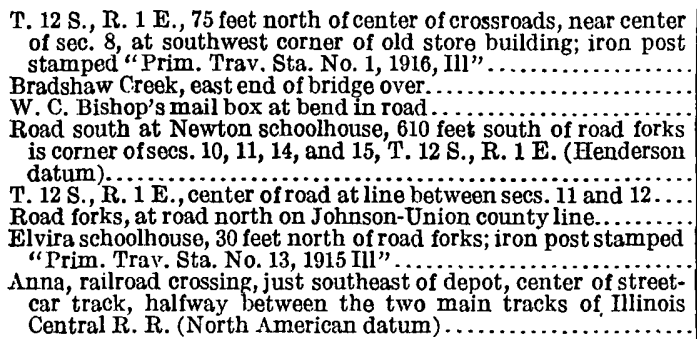 & $\begin{array}{lll}37 & 29 & 00.1 \\
37 & 29 & 06.9 \\
37 & 29 & 10.4 \\
37 & & \\
37 & 36.9 \\
& & \\
37 & 27 & 36.7\end{array}$ & $\begin{array}{lll}89 & 04 & 48.6 \\
89 & 03 & 43.1 \\
89 & 02 & 36.9 \\
& & 1 \\
89 & 01 & 49.5\end{array}$ & $\begin{array}{r}\text { Fect. } \\
\cdots 3,611 \\
4,541 \\
\\
5,166 \\
5,320 \\
5,340\end{array}$ \\
\hline
\end{tabular}

West along highways through center of quadrangle.

T road east (Henderson datum) .....................................
T.13\$. R. 2 E., quarter corner, west side of sec. 30 , Union-Johnson county líne.

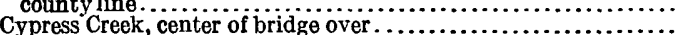

T. 13 S., R. 1 E., line between secs. 15 and 22 , center of road in line with fence east, 0.5 mile south of Friendship Church

T. 13 S., R. 1 E., quarter corner between secs. 16 and 21 , at bend

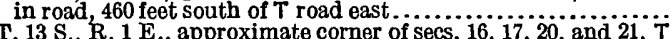

T. 13 S., R 1 approximate corner of secs, $16,17,20$, and $21, T$

road south at Karraker schoolhouse.................................

Karraker schoolhouse, 0.75 mile west of, on embankment at
northeast corner of $T$ road north; iron post stamped "Prim. Trav. Sta. No. 2, 1916 Ill".

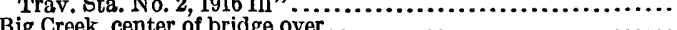

Third Principal Meridian (approximate) center of roa

Dongola, Baggott's grocery and furniture store, southwest corner of (approximate North A merican datum).............................. line follows road north

$T$ road west.

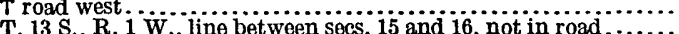

Oak tree at road forks at road south . .........................

Road west, at Chapel Church.

St. John's Church, 0.3 mile west of $\mathrm{T}$ road east. . . .

T road west.

\begin{tabular}{|c|c|c|}
\hline 372101.5 & $\begin{array}{lll}89 & 01 & 48.2\end{array}$ & \\
\hline $\begin{array}{lll}37 & 21 & 09.5 \\
37 & 21 & 42.4\end{array}$ & $\begin{array}{llll}89 & 02 & 39.3 \\
89 & 04 & 06.3\end{array}$ & $\begin{array}{l}4,204 \\
7,775\end{array}$ \\
\hline 372237.7 & $\begin{array}{llll}89 & 05 & 40.4\end{array}$ & 9,442 \\
\hline $\begin{array}{lll}37 & 22 & 41.1\end{array}$ & $8906 \quad 29.4$ & 3,965 \\
\hline $\begin{array}{llll}37 & 22 & 40.9\end{array}$ & $\begin{array}{lll}89 & 07 & 02.4\end{array}$ & 2,660 \\
\hline $\begin{array}{lll}37 & 22 & 36.6 \\
37 & 22 & 30.5 \\
37 & 22 & 08.3\end{array}$ & $\begin{array}{lll}89 & 07 & 50.8 \\
89 & 08 & 15.6 \\
89 & 09 & 24.0\end{array}$ & $\begin{array}{l}3,930 \\
2,080 \\
5,952\end{array}$ \\
\hline $\begin{array}{lll}37 & 21 & 44.4\end{array}$ & $\begin{array}{llll}89 & 09 & 56.2\end{array}$ & 3,540 \\
\hline $\begin{array}{lll}37 & 22 & 25.4 \\
37 & 22 & 51.8 \\
37 & 22 & 56.2 \\
37 & 22 & 59.2 \\
37 & 23 & 14.0 \\
37 & 23 & 14.8 \\
37 & 22 & 41.8\end{array}$ & $\begin{array}{lll}89 & 11 & 03.8 \\
89 & 11 & 53.9 \\
89 & 12 & 44.0 \\
89 & 13 & 05.0 \\
89 & 13 & 59.4 \\
89 & 14 & 58.6 \\
89 & 14 & 59.6\end{array}$ & $\begin{array}{l}6,860 \\
4,849 \\
4,064 \\
1,726 \\
4,639 \\
4,770 \\
3,325\end{array}$ \\
\hline
\end{tabular}

East along Chicago \& Eastern Illinois R. R. and bighways near south border of quadrangle

T. 14 S., R. 1 W., railroad crossing at east and west road probably

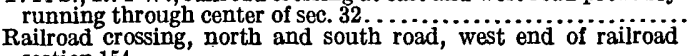

Railroad crossing, 300 feet southwest of milepost 20 (mean of

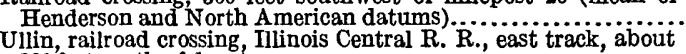
300 feet south of depot

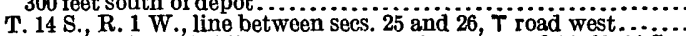
Third Principal Meridian, west corner of secs. 19 and 30 , T. 14 S.,

R. 1 E., lane north, roads east and south.

$T$ road west

Good Hope Church, 15 feet south of line between secs. 29 and 32 ,

T. 14 S., R. 1 E., at southeast corner of T road east; iron post stamped "Prim. Trav. Sta. No. 7, 1916 Ill" .................. Crossroads.

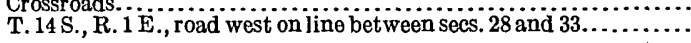

\begin{tabular}{|c|c|c|}
\hline $\begin{array}{lll}37 & 15 & 15.8\end{array}$ & 891424.8 & $\ldots \ldots \ldots$ \\
\hline $\begin{array}{lll}37 & 15 & 51.8\end{array}$ & 891316.1 & 6,619 \\
\hline $\begin{array}{lll}37 & 16 & 26.8\end{array}$ & 891155.6 & 7,390 \\
\hline $\begin{array}{lll}37 & 16 & 34.4 \\
37 & 16 & 14.0\end{array}$ & $\begin{array}{lll}89 & 11 & 06.2 \\
89 & 10 & 29.8\end{array}$ & $\begin{array}{l}4,062 \\
3,594\end{array}$ \\
\hline $\begin{array}{lll}37 & 16 & 18.1 \\
37 & 16 & 16.2\end{array}$ & $\begin{array}{lll}89 & 09 & 25.1 \\
89 & 07 & 54.4\end{array}$ & $\begin{array}{l}5,242 \\
7,320\end{array}$ \\
\hline $\begin{array}{lll}37 & 15 & 23.4 \\
37 & 15 & 22.0 \\
37 & 15 & 21.2\end{array}$ & $\begin{array}{lll}89 & 07 & 54.4 \\
89 & 06 & 48.4 \\
89 & 06 & 03.5\end{array}$ & $\begin{array}{l}5,361 \\
5,330 \\
3,620\end{array}$ \\
\hline
\end{tabular}

Magnetic declination along north border of quadrangle, $5^{\circ} \mathrm{E}$; ; through center of quadrangle, $4^{\circ} 40^{\prime} \mathrm{E}$.; along south border of quadrangle, $4^{\circ} 30^{\prime} \mathrm{E}$. 
GENOA QUADRANGLE.

South along highways near west border of quadrangle. (By F. W. Crisp in 1917.)

\begin{tabular}{|c|c|c|c|}
\hline Station. & Latitude. & Longitude. & $\begin{array}{l}\text { Distance } \\
\text { between } \\
\text { stations. }\end{array}$ \\
\hline 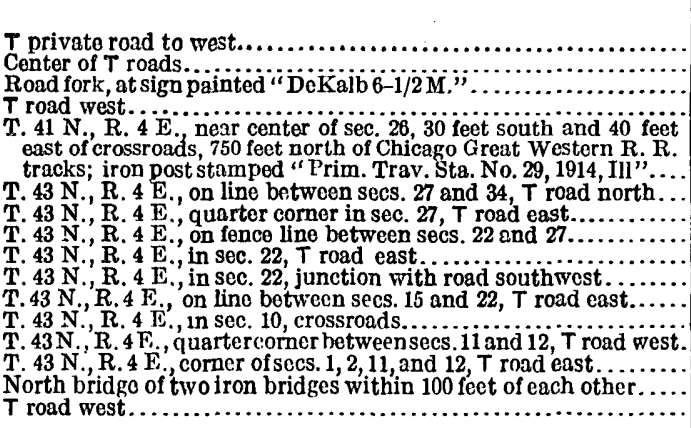 & $\begin{array}{lcc}0 & \prime \prime & \prime \prime \\
42 & 02 & 09.0 \\
42 & 01 & 41.9 \\
42 & 01 & 31.2 \\
42 & 01 & 12.4 \\
& & \\
42 & 00 & 09.7 \\
42 & 10 & 05.5 \\
42 & 10 & 31.6 \\
42 & 10 & 57.7 \\
42 & 11 & 10.9 \\
42 & 11 & 42.9 \\
42 & 11 & 50.0 \\
42 & 13 & 08.4 \\
42 & 13 & 08.6 \\
42 & 13 & 26.6 \\
42 & 14 & 01.4 \\
42 & 14 & 27.5\end{array}$ & $\begin{array}{ccc}\circ & \prime & \prime \prime \\
88 & 44 & 49.4 \\
88 & 44 & 39.2 \\
88 & 44 & 35.4 \\
88 & 44 & 34.7 \\
& & \\
88 & 44 & 23.6 \\
88 & 44 & 56.9 \\
88 & 44 & 56.9 \\
88 & 44 & 56.8 \\
88 & 44 & 56.7 \\
88 & 44 & 56.8 \\
88 & 44 & 56.8 \\
88 & 44 & 57.5 \\
88 & 43 & 29.2 \\
88 & 43 & 29.4 \\
88 & 43 & 29.4 \\
88 & 43 & 29.4\end{array}$ & $\begin{array}{r}\text { Feet. } \\
\ldots \ldots \\
2,840 \\
1,127 \\
1,902 \\
\\
6,701 \\
\ldots \ldots 9 \\
2,650 \\
2,640 \\
1,330 \\
3,240 \\
720 \\
\ldots, 934 \\
6,644 \\
1,820 \\
3,520 \\
2,640\end{array}$ \\
\hline
\end{tabular}

Magnetic declination along north half of west border of quadrangle, $3.3^{\circ} \mathrm{E}$.

HARVARD QUADRANGLE.

Along hlghways near southwest corner of quadrangle. (By F. W. Crisp in 1017.)

\begin{tabular}{|c|c|c|c|}
\hline Station. & Latitude. & Longitude. & $\begin{array}{l}\text { Distance } \\
\text { between } \\
\text { stations. }\end{array}$ \\
\hline 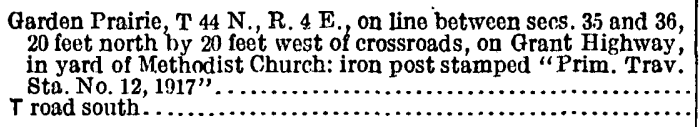 & $\begin{array}{ccc}\circ & \prime & \prime \prime \\
42 & 15 & 12.6 \\
42 & 15 & 14.8\end{array}$ & $\begin{array}{ccc}\circ & \prime & \prime \prime \\
88 & 43 & 29.7 \\
88 & 44 & 40.7\end{array}$ & $\begin{array}{c}\text { Fcct. } \\
\cdots, \ldots, 340\end{array}$ \\
\hline
\end{tabular}

Magnetic declination near southwest corner of quadrangle, $3.3^{\circ} \mathrm{E}$.

Worth along highways near west border of quadrangle. (By F. W. Crisp in 1917.)

Garden Prairie station, 600 feet west of, road crossing............ T. 44 N., R. 4 E., quarter corner between sees. 25 and $26, \%$ road

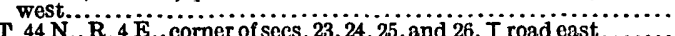

T. 44 N., R. 4 E., quarter corner between secs. 23 and 24 , crossroads

T. 44 N., R. 4 E., quarter corner between secs. 23 and 24 , crossroads.

T. 44 N., R. 4 E., corner of sees. $13,14,23$, and 24 , fence line.........

T. 44 N., R 4 E., corner of secs. 11, 12, 13, and 14, fence line.

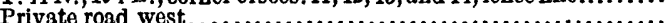

T. 44 N., R. 4 E., corner ofsecs. $1,2,11$, and i2, crossroads at school-

house.

$T$ road south

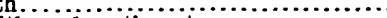

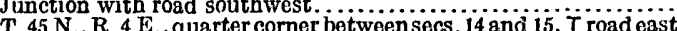

T. 45 N., R. 4 E., corner of secs. $10,11,14$, and $15, T$ road west.

$\mathrm{T} .45 \mathrm{~N}$., $\mathrm{R} .4$ E., quarter corner between secs. 10 and $11, \mathrm{~T}$ road

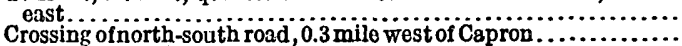

T. 45 N., R. 4 E. quarter corner between secs. 2 and 3 crossroads.

Tps. 45 and 46 N., R. 4 E., corner of secs. $34,35,2$, and 3 , fence...

T. 46 N., R. 4 E., quarter corner between secs. 34 and 35 , fence line.

T. 46 N., R. 4 E., section line between secs. 27 and 34, crossroads.

$T$ road west.

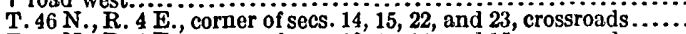

T. 46 N., R. 4 E., corner of secs. $10,11,14$, and 15 , crossroads.....

T. 46 N., R. 4 E., north side, corner of secs. 2 and 3 , on Illinois-

Wisconsin Stateline, $T$ road south

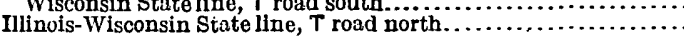

\begin{tabular}{|c|c|c|}
\hline $\begin{array}{lll}42 & 15 & 16.4\end{array}$ & $\begin{array}{lll}88 & 43 & 29.3\end{array}$ & \\
\hline $\begin{array}{lll}42 & 15 & 45.8 \\
42 & 16 & 11.9 \\
42 & 16 & 37.8 \\
42 & 17 & 04.0 \\
42 & 17 & 30.1 \\
42 & 17 & 56.2 \\
42 & 18 & 22.3\end{array}$ & $\begin{array}{lll}88 & 43 & 29.7 \\
88 & 43 & 29.7 \\
88 & 43 & 29.7 \\
88 & 43 & 29.7 \\
88 & 43 & 29.9 \\
88 & 43 & 30.0 \\
88 & 43 & 30.1\end{array}$ & $\begin{array}{l}2,980 \\
2,640 \\
2,630 \\
2,650 \\
2,640 \\
2,650 \\
2,640\end{array}$ \\
\hline $\begin{array}{lll}42 & 18 & 48.3 \\
42 & 18 & 47.9 \\
42 & 22 & 37.6 \\
42 & 22 & 43.7 \\
42 & 23 & 09.8\end{array}$ & $\begin{array}{lll}88 & 43 & 30.3 \\
88 & 44 & 49.1 \\
88 & 44 & 40.6 \\
88 & 44 & 40.6 \\
88 & 44 & 40.8\end{array}$ & $\begin{array}{r}2,640 \\
5,920 \\
\ldots \ldots \\
620 \\
2,640\end{array}$ \\
\hline $\begin{array}{lll}42 & 23 & 35.8 \\
42 & 23 & 44.1 \\
42 & 23 & 55.4 \\
42 & 24 & 28.1 \\
42 & 24 & 53.4 \\
42 & 25 & 10.5 \\
42 & 25 & 45.6 \\
42 & 26 & 30.0 \\
42 & 27 & 30.2 \\
42 & 28 & 22.3\end{array}$ & $\begin{array}{lll}88 & 44 & 40.9 \\
88 & 44 & 41.0 \\
88 & 44 & 41.0 \\
88 & 44 & 41.2 \\
88 & 44 & 41.6 \\
88 & 44 & 41.8 \\
88 & 44 & 45.7 \\
88 & 44 & 41.9 \\
88 & 44 & 41.9 \\
88 & 44 & 42.5\end{array}$ & $\begin{array}{l}2,640 \\
840 \\
1,150 \\
3,310 \\
2,560 \\
2,650 \\
2,655 \\
4,508 \\
6,100 \\
5,270\end{array}$ \\
\hline & $\begin{array}{lll}88 & 44 & 45.4 \\
88 & 44 & 18.9\end{array}$ & 7,12 \\
\hline
\end{tabular}

Magnetic declination along west border of quadrangle, $3^{\circ} \mathrm{E}$. $121166^{\circ}-23-$ Bull. $709-12$ 
HAVANA QUADRANGLE.

North along hlghways near west border of quadrangle. (By J. H. Wilson in 1916.)

\begin{tabular}{|c|c|c|c|}
\hline Station. & Latitude., & Longitude. & $\begin{array}{l}\text { Distance } \\
\text { between } \\
\text { stations. }\end{array}$ \\
\hline 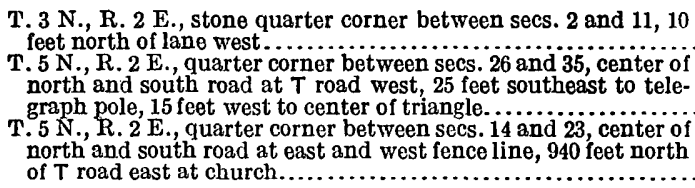 & $\begin{array}{l}\circ \quad, \quad \prime \prime \\
40 \quad 1535.8 \\
402243.4 \\
402427.8\end{array}$ & $\begin{array}{ccc}\circ & \prime & \prime \prime \\
90 & 14 & 52.9 \\
90 & 14 & 44.1 \\
90 & 14 & 42.3\end{array}$ & $\begin{array}{c}\text { Feet. } \\
\ldots \ldots \ldots\end{array}$ \\
\hline
\end{tabular}

Magnetic declination for west border of quadrangle, $5^{\circ} 12^{\prime} \mathrm{E}$.

\section{JONESBORO QUADRANGLE.}

North along Moblle \& Ohlo and Illinois Central railroads near east border of quadrangle. (By J. H. Wilson in 1916.)

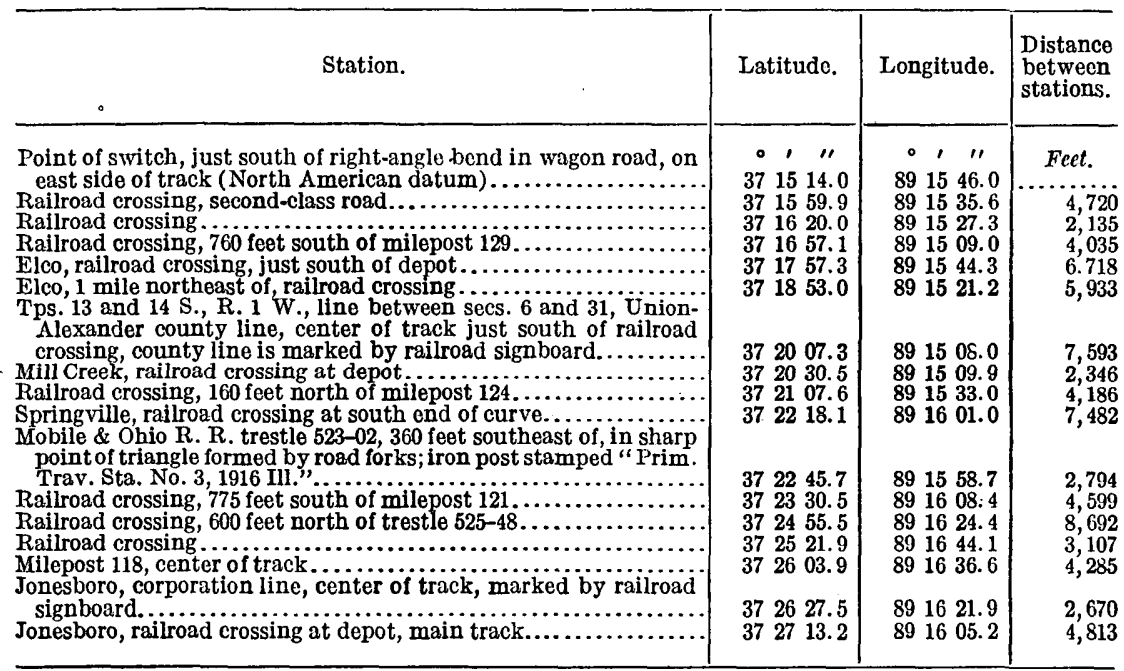

North along Illinols Central R. R.

\begin{tabular}{|c|c|c|c|}
\hline $\begin{array}{l}\text { Railroad crossing, between tracks............................. } \\
\text { Railroad crossing, northbound track }\end{array}$ & $\begin{array}{lll}37 & 28 & 10.4 \\
37 & 29 & 00.6\end{array}$ & $\begin{array}{lll}89 & 15 & 23.4 \\
89 & 15 & 34.3\end{array}$ & 5,1 \\
\hline 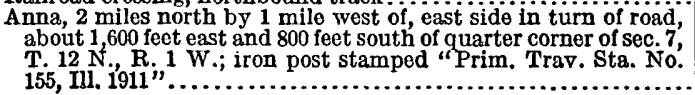 & 372920.0 & $\begin{array}{lll}89 & 15 & 50.7\end{array}$ & 2,366 \\
\hline
\end{tabular}

North along Illinots Central R. R. near Mississippi River.

Railroad crossing (North American datum)

Railroad crossing 460 feet north of milepost 123 ........

T. 14 S., R. 3 W., line between secs. 21 and 28 , center of track. .

T. 14 S., R. 3 W., line through center of sec. 16, east and west railroad crossing, 440 feet south of milepost 121 .

T. 14 S., R. 3 W., line between secs. 9 and 16 , center of track, 275

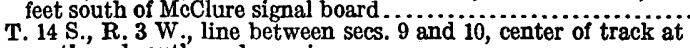
north and south read crossing ................................ Tps. 13 and $14 \mathrm{~N}$, line between secs. 2 and 35, Alexander-Union county line center of Illinois Central R. R. track, just west of signboard along Missouri Pacific R. R. track..................

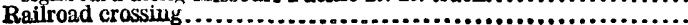

\begin{tabular}{|c|c|c|}
\hline $\begin{array}{lll}37 & 16 & 00.5 \\
37 & 16 & 21.8 \\
37 & 16 & 39.1\end{array}$ & $\begin{array}{lll}89 & 26 & 22.8 \\
89 & 26 & 22.5 \\
89 & 26 & 22.2\end{array}$ & $\begin{array}{r}\because, 1600 \\
1,750\end{array}$ \\
\hline $\begin{array}{lll}37 & 17 & 57.3\end{array}$ & 892621.1 & 7,905 \\
\hline $\begin{array}{lll}37 & 18 & 23.8\end{array}$ & $\begin{array}{lll}89 & 26 & 20.7\end{array}$ & 2,675 \\
\hline $\begin{array}{lll}37 & 18 & 56.4 \\
37 & 19 & 05.6\end{array}$ & $\begin{array}{llll}89 & 26 & 03.3 \\
89 & 25 & 44.8\end{array}$ & $\begin{array}{l}3,589 \\
1,760\end{array}$ \\
\hline $\begin{array}{lll}37 & 20 & 04 . \\
37 & 20 & 55 .\end{array}$ & $\begin{array}{lll}89 & 24 & 34.0 \\
89 & 24 & 05.1\end{array}$ & $\begin{array}{l}8,2 \\
5,6\end{array}$ \\
\hline
\end{tabular}


JONESBORO QUADRANGLE-Continued.

North along Illinois Central R. R. near Mississippi River-Continued.

\begin{tabular}{|c|c|c|c|}
\hline Station. & Latitude. & Longitude. & $\begin{array}{l}\text { Distance } \\
\text { between } \\
\text { stations. }\end{array}$ \\
\hline 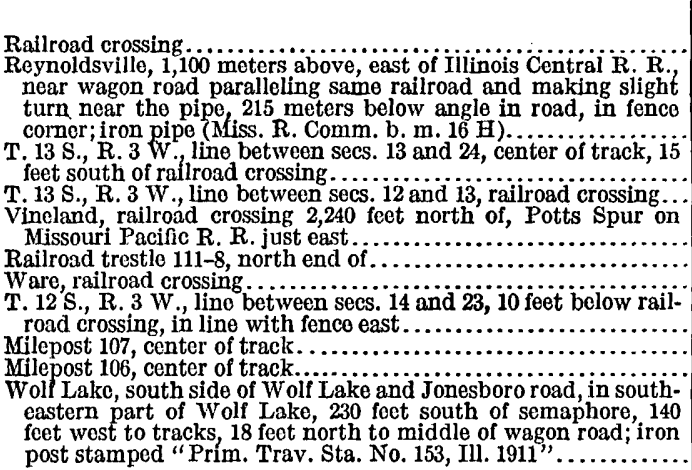 & $\begin{array}{lll}37 & 22 & 34.55 \\
37 & 22 & 41.2 \\
37 & 23 & 34.2 \\
& & \\
37 & 24 & 36.0 \\
37 & 25 & 12.4 \\
37 & 26 & 51.7 \\
& & \\
37 & 27 & 58.3 \\
37 & 29 & 04.0 \\
37 & 29 & 46.5\end{array}$ & $\begin{array}{l}8923 \text { 54.0 } \\
892321.61 \\
892320.8 \\
892319.0 \\
892319.1 \\
8923 \\
8923.3 \\
8924.6 \\
892508.7 \\
8925,46.7\end{array}$ & $\begin{array}{r}7,800 \\
\\
670 \\
5,369 \\
6,245 \\
3,699 \\
10,195 \\
7,050 \\
8,129 \\
5,280\end{array}$ \\
\hline
\end{tabular}

West along highways through conter of quadrangle.

T. 13 S., R. 2 W., T road north through center of sec. 13.

Misenheimer schoolhouse, about 300 feet northwest of, on hili,

front gate of Dillon's residence............................

Second-class road southwest.

Mail box $84-A$, in front of house on south side of road, 460 feet

$T$ road east, at southeast corner of, 0.5 mile south of schoolhouse; iron post stamped "Prim. Trav.'Sta. No. 4, 1916 Ill." ..........

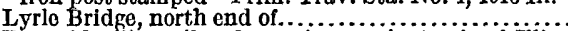

Reynoldsvllie, railroad crossing, main track of illinois Contra] R. R.

\begin{tabular}{|rrr|rrr|r}
37 & 22 & 53.4 & 89 & 16 & 44.6 & $\ldots \ldots$ \\
37 & 23 & 04.5 & 89 & 17 & 50.1 & \\
37 & 23 & 35.7 & 89 & 18 & 17.7 & 5,414 \\
37 & 23 & 22.0 & 89 & 19.58 .6 & 3,867 \\
37 & 22 & 09.7 & 89 & 21 & 10.0 & 8,260 \\
37 & 22 & 07.2 & 89 & 21 & 30.0 & 9,316 \\
37 & 22 & 01.7 & 89 & 23 & 37.3 & 1,633 \\
& & & & \\
\end{tabular}

Magnetic declination through center of quadrangle, $4^{\circ} \cdot 35^{\prime} \mathrm{E}$.

ETHGS QUADRANGLE.

West along highways near north border of quadrangle. (By F. W. Crisp in 1917.)

\begin{tabular}{|c|c|c|c|}
\hline Station. & Latitude. & Longitude. & $\begin{array}{l}\text { Distance } \\
\text { between } \\
\text { stations. }\end{array}$ \\
\hline 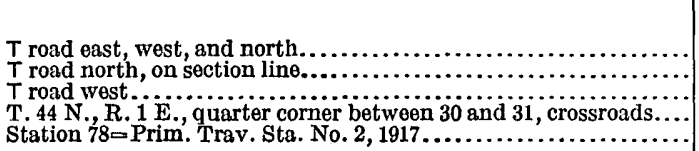 & $\begin{array}{ccc}\circ & , & \prime \prime \\
42 & 14 & 56.7 \\
42 & 14 & 54.1 \\
42 & 14 & 41.6 \\
42 & 14 & 41.1 \\
42 & 14 & 40.3\end{array}$ & $\begin{array}{ccc}\circ & \prime & \\
89 & 06 & 56.8 \\
89 & 08 & 04.2 \\
89 & 08 & 29.0 \\
89 & 08 & 17.5 \\
89 & 10 & 23.2\end{array}$ & \begin{tabular}{|r} 
Fect. \\
$\mathbf{5}, 074$ \\
2,259 \\
3,650 \\
4,941
\end{tabular} \\
\hline
\end{tabular}

West along highways near north border of quadrangle.

T. 44 N., R. 1 E., quarter corner between secs. 31 and 30 , principal meridian, 35 feet south and 30 feet west of $T$ road south, center of crossroads; iron post stamped "Prim. Trav. Sta. No.2, 1917" T. 26 N., R. 11 E., quarter corner between secs. 14 and 23 , center

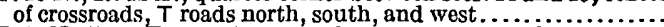
T. 26 N., R. 11 E., quarter corner between secs. 15 and 22, center of crossroad, $T$ road north.

T. 26 N., $R$. 11 E., corner of secs. $15,16,21$, and 22 , fence to south. T. 26 N., R. 11 E., quarter corner between secs. 16 and $21, T$ road

T. 26 N., R. 11 E., corner of secs. $16,17,21$, and $22, \mathrm{~T}$ road north, center of crossroad.
421440.3

421452.2

421451.3

$42 \quad 1450.9$

421450.5

.421450 .4
891023.2

891132.1

891241.9

891317.0

891351.8

891426.8
5,319

5,250

2,640

2,620

2,630 
KINGS QUADRANGLE-Continued.

South along highways on third principal meridian through quadrangle. (By F. W. Crisp in 1917.)

\begin{tabular}{|c|c|c|c|}
\hline Station. & Latitude. & Longitude. & $\begin{array}{l}\text { Distance } \\
\text { between } \\
\text { stations. }\end{array}$ \\
\hline & 0,11 & 0,11 & Fect. \\
\hline 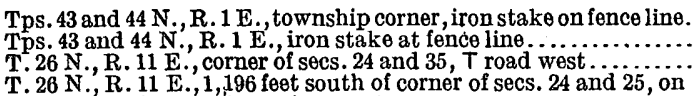 & $\begin{array}{lll}42 & 14 & 14.4 \\
42 & 14 & 14.4 \\
42 & 14 & 00.7\end{array}$ & $\begin{array}{lll}89 & 10 & 22.2 \\
89 & 10 & 22.2 \\
89 & 10 & 22.4\end{array}$ & $\begin{array}{r}2,619 \\
1,390\end{array}$ \\
\hline 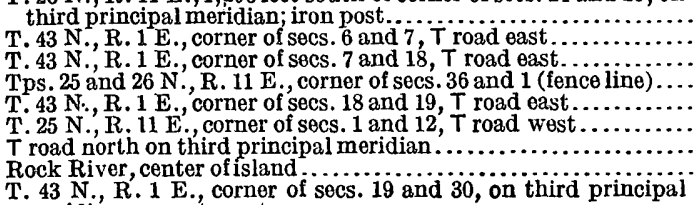 & $\begin{array}{lll}42 & 13 & 48.8 \\
42 & 13 & 23.3 \\
42 & 12 & 31.0 \\
42 & 12 & 16.2 \\
42 & 11 & 38.6 \\
42 & 11 & 19.0 \\
42 & 10 & 58.9 \\
42 & 10 & 51.1\end{array}$ & $\begin{array}{llll}89 & 10 & 21.9 \\
89 & 10 & 22.0 \\
89 & 10 & 21.7 \\
89 & 10 & 21.7 \\
89 & 10 & 21.6 \\
89 & 10 & 21.4 \\
89 & 10 & 21.4 \\
89 & 10 & 21.3\end{array}$ & $\begin{array}{r}1,196 \\
2,581 \\
5,300 \\
1,490 \\
3,810 \\
1,980 \\
2,040 \\
785\end{array}$ \\
\hline 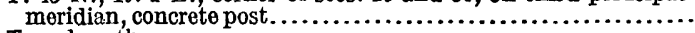 & 421044.1 & 891021.2 & 707 \\
\hline 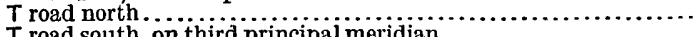 & 420946.7 & 891005.7 & 5,923 \\
\hline 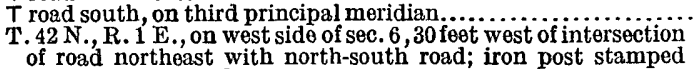 & 420939.2 & 891021.2 & 1,453 \\
\hline 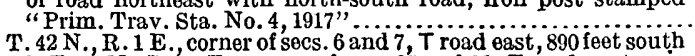 & 420842.8 & 891021.0 & 5,610 \\
\hline is $\mathrm{T} .25 \mathrm{~N} ., \mathrm{R} .11 \mathrm{E} .$, corner of secs. 25 and $36, \mathrm{~T}$ road west...... & 420809.2 & 891020.5 & 3,392 \\
\hline 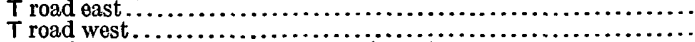 & $\begin{array}{l}420717.3 \\
420652.3\end{array}$ & $\begin{array}{lll}89 & 10 & 32.1 \\
89 & 10 & 42.8\end{array}$ & $\begin{array}{l}5,326 \\
2,660\end{array}$ \\
\hline $\begin{array}{l}\text { Road forks, roads north, south, and southeast . } \\
\text { Stillmans Valley railway station, } 800 \text { feet west of, at crossing of }\end{array}$ & 420640.3 & 891050.6 & 1,343 \\
\hline public road and Chicago, Milwaukee \& St. Paul $\mathbf{R y} . \ldots \ldots \ldots \ldots$ & 420636.4 & 891053.2 & 445 \\
\hline 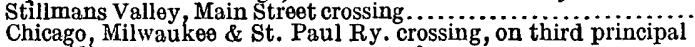 & 420629.2 & 891043.2 & 1,046 \\
\hline 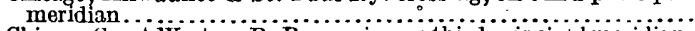 & 420629.2 & 891020.2 & 1,740 \\
\hline 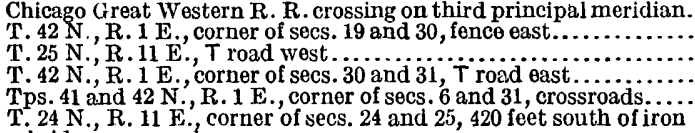 & $\begin{array}{lll}42 & 06 & 13.5 \\
42 & 05 & 33.2 \\
42 & 05 & 06.1 \\
42 & 04 & 41.1 \\
42 & 03 & 48.1\end{array}$ & $\begin{array}{lll}89 & 10 & 20.0 \\
89 & 10 & 20.4 \\
89 & 10 & 20.5 \\
89 & 10 & 20.6 \\
89 & 10 & 20.5\end{array}$ & $\begin{array}{l}1,589 \\
4,080 \\
2,740 \\
2,535 \\
5,365\end{array}$ \\
\hline 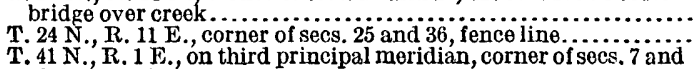 & $\begin{array}{lll}42 & 03 & 28.3 \\
42 & 02 & 42.9\end{array}$ & $\begin{array}{lll}89 & 10 & 20.2 \\
89 & 10 & 19.2\end{array}$ & $\begin{array}{l}2,000 \\
4,600\end{array}$ \\
\hline T. 24 , N., R. 11 E., corner of fractional secs. 1 and 36 , on third prin- & 420205.4 & 891018.4 & 3,790 \\
\hline 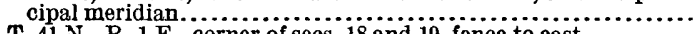 & 420150.0 & 891018.0 & 1,560 \\
\hline T. 41 N., R. 1 E., corner of secs. 18 and 19 , fence to east....... & 420113.0 & 891017.2 & 3,750 \\
\hline 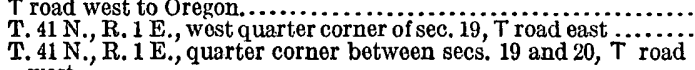 & $\begin{array}{l}420059.1 \\
4200 \quad 46.5\end{array}$ & 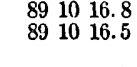 & $\begin{array}{l}1,400 \\
1,280\end{array}$ \\
\hline 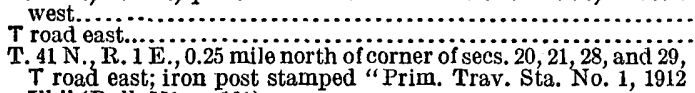 & $\begin{array}{l}420047.0 \\
420033.9\end{array}$ & $\begin{array}{lll}89 & 09 & 10.7 \\
89 & 09 & 10.2\end{array}$ & $\begin{array}{l}4,973 \\
1,320\end{array}$ \\
\hline 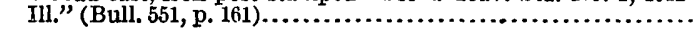 & 420034.8 & 890800.6 & 5,253 \\
\hline
\end{tabular}

South along highways near east border of quadrangle.

T. 41 N., R. 2 E., north corner of secs, 3 and 4, on township line,

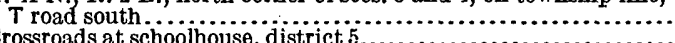
Crossroads at schoolhouse, district 5 .

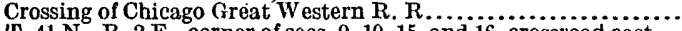

T. 41 N., R. 2 E., corner of secs. $9,10,15$, and 16 , crossroad east....

T. 41 N., R. 2 E., corner of secs. 15, 16, 21, and 22, crossroads, macadam east to west.

T. 41 N., R. 2 E., 20 feet south by 25 feet west of corner of secs. 21 , 22,27 , and 28 , at $T$ road corners; iron post stamped "Prim. Trav.'Sta. No. 2, 1912"

\begin{tabular}{|c|c|c|}
\hline $\begin{array}{lll}42 & 03 & 52.9 \\
42 & 03 & 20.6 \\
42 & 02 & 40.7 \\
42 & 02 & 11.4\end{array}$ & $\begin{array}{llll}89 & 00 & 00.1 \\
89 & 00 & 00.4 \\
89 & 00 & 00.7 \\
89 & 00 & 01.5\end{array}$ & $\begin{array}{r}3,270 \\
4,040 \\
2,960\end{array}$ \\
\hline $4201 \quad 19.2$ & $\begin{array}{lll}89 & 00 & 01.8\end{array}$ & 5,280 \\
\hline 420026.9 & 890001.8 & 5,296 \\
\hline
\end{tabular}

East along highways through center of quadrangle.

Prim. Trav. Sta. No. 4, 1917 (previously described)

T. 41 N., R. 1 E., quarter corner, north side of sec. $5, T$ road to

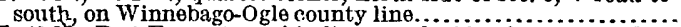
T. 42 N., R. 1 E., on township line, south side of secs. 32 and 33

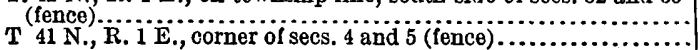

420842.8

420901.6

420901.9

420902.0
891021.0

$\begin{array}{lll}89 & 08 & 35.7\end{array}$

89.0804 .9

89.0802 .4
8,157

2, 320 
KINGS QUTADRANGLE-Continued.

East along highways through center of quadrangle-Continued.

\begin{tabular}{|c|c|c|c|}
\hline Station. & Latitude. & Longitude. & $\begin{array}{l}\text { Distance } \\
\text { between } \\
\text { stations. }\end{array}$ \\
\hline 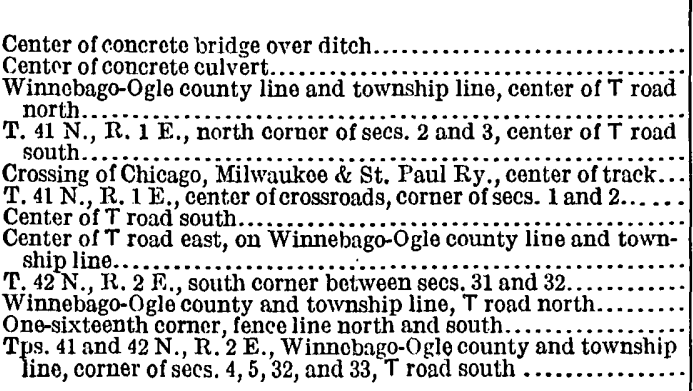 & $\begin{array}{ccc}\circ & \prime \prime & \prime \prime \\
42 & 09 & 02.1 \\
42 & 09 & 02.5 \\
42 & 09 & 02.8 \\
42 & & \\
42 & 09 & 03.0 \\
42 & 09 & 03.1 \\
42 & 09 & 03.5 \\
42 & 09 & 03.9 \\
42 & 09 & 04.1 \\
42 & 09 & 01.9 \\
42 & 09 & 05.1 \\
42 & 09 & 05.6 \\
42 & 09 & 05.8\end{array}$ & \begin{tabular}{ccc}
$\circ$ & \multicolumn{1}{c}{ " } \\
89 & 07 & 52.8 \\
89 & 06 & 53.7 \\
89 & 06 & 02.2 \\
& & \\
89.05 & 46.7 \\
89.05 & 29.6 \\
89.04 & 36.3 \\
89 & 03 & 41.0 \\
89 & 03 & 11.7 \\
89.02 & 13.4 \\
89.01 & 56.0 \\
89 & 01 & 21.0 \\
89 & 01 & 03.2
\end{tabular} & $\begin{array}{r}\text { Feet. } \\
720 \\
4,445 \\
3,878 \\
1,170 \\
1,290 \\
4,020 \\
4,160 \\
2,214 \\
4,390 \\
1,310 \\
2,640 \\
1,340\end{array}$ \\
\hline
\end{tabular}

Magnetic declination along third principal meridian, $4.1^{\circ} \mathrm{E}$.

KIRKIAND QUADRANGLE.

South along highways near west border of quadrangle. (By F. W. Crisp in 1917.)

\begin{tabular}{|c|c|c|c|}
\hline Station. & Latitude. & Longitude. & $\begin{array}{l}\text { Distance } \\
\text { between } \\
\text { stations. }\end{array}$ \\
\hline 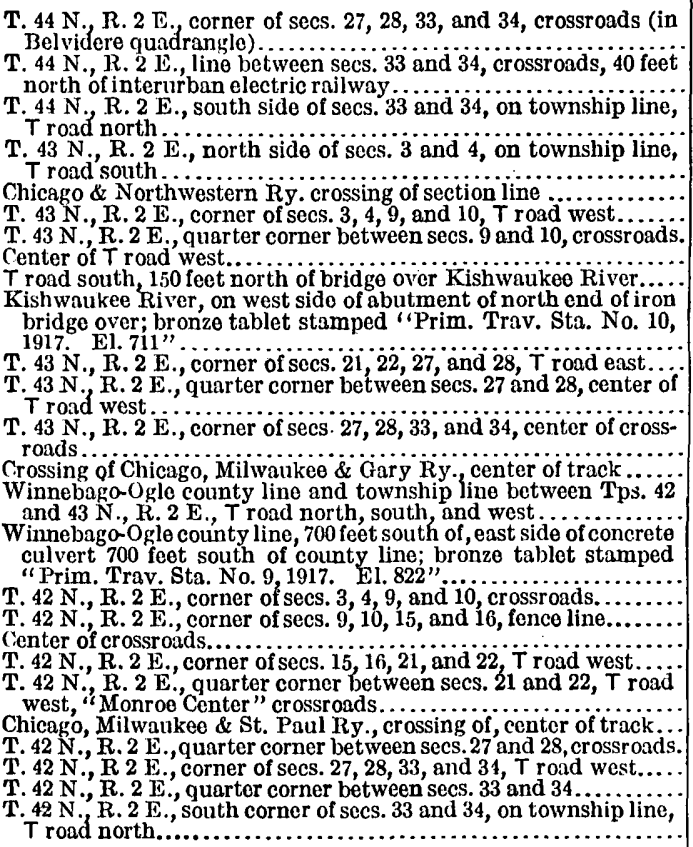 & $\begin{array}{ccc}\circ & \prime \prime & \prime \prime \\
42 & 15 & 12.0 \\
42 & 14 & 41.2 \\
42 & 14 & 19.6 \\
42 & 14 & 19.6 \\
42 & 13 & 43.0 \\
42 & 13 & 28.3 \\
42 & 13 & 02.3 \\
42 & 12 & 23.1 \\
42 & 11 & 43.9\end{array}$ & \begin{tabular}{ccc}
$\circ$ & $\prime$ & \multicolumn{1}{l}{} \\
88 & 59 & 57.8 \\
88 & 59 & 57.7 \\
88 & 59 & 57.7 \\
& & \\
88 & 59 & 55.3 \\
88 & 59 & 54.7 \\
88 & 59 & 54.6 \\
88 & 59 & 54.4 \\
88 & 59 & 54.2 \\
88 & 59 & 56.6
\end{tabular} & $\begin{array}{r}\text { Feet. } \\
\ldots \ldots \ldots \\
3,120 \\
2,180 \\
\\
180 \\
3,710 \\
1,480 \\
2,930 \\
3,970 \\
3,972\end{array}$ \\
\hline
\end{tabular}


KIRKILAND QUADRANGLE-Continued.

East along highways through center of quadrangle.

\begin{tabular}{|c|c|c|c|}
\hline Station. & Latitude. & Longitude. & $\begin{array}{l}\text { Distance } \\
\text { between } \\
\text { stations. }\end{array}$ \\
\hline $\begin{array}{l}\text { Junction with line north and south, Winnebago-Ogle county line } \\
\text { and township line between Tps. } 42 \text { and } 43 \mathrm{~N} . \mathrm{R} .2 \mathrm{E} . \text {, corner of } \\
\text { secs. } 3,4,33 \text {, and } 34, \mathrm{~T} \text { road north, south, and west........... }\end{array}$ & $\begin{array}{ccc}\circ & \prime \prime \\
42 & 09 & 06.6\end{array}$ & $\begin{array}{ccc}\circ & \prime \prime & \prime \prime \\
88 & 59 & 52.7\end{array}$ & $\begin{array}{l}\text { Feet. } \\
5,309\end{array}$ \\
\hline
\end{tabular}

Magnetic declination along east and west borders of quadrangle, $3.7^{\circ} \mathrm{E}$.; through center of quadrangle, $3.7^{\circ} \mathrm{E}$.

Winnebago-Ogle county line, Tps. 42 and 43 N., R. 2 E., corner

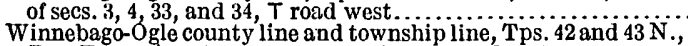
R. 2 E., corner of secs. $2,3,34$, and 35 , T road east.............. $T$ road south

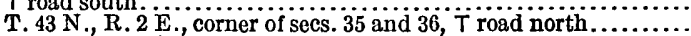

Tps. 42 and 43 N., Rs. 2 and 3 E., T road west.

T. 43 N., R. 3 E., quarter corner of sec. 31 , Winnebago-Boone

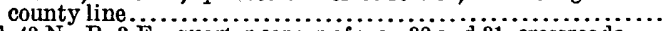
T. 43 N., R. 3 E., quarter corner of secs. 30 and 31 , crossroads..... T. 43 N., R. 3 E., quarter corner between secs. 29 and 30 , cross-

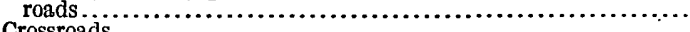

Crossroads.........

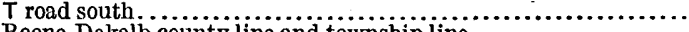

Boone-Dekalb county line and township line......................

T road south

Charter Oak School, district 24 , on north-south section line, cross-

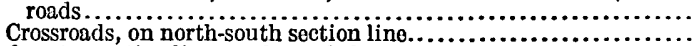

Quarter-section line north-south fence.................................

North-south section-line fence.

T road north at church .............................................

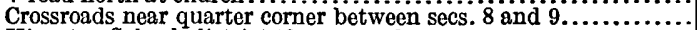

Kingston School, district 12, crossroads .

420906.6

420907.6 420908.0 420908.2

420909.0

420935.2 420935.6

420936.6 $\begin{array}{lll}42 & 09 & 37.4\end{array}$ 420933.4 $\begin{array}{lll}42 & 09 & 11.4\end{array}$ 420848.4 $42 \quad 0826.9$

$\begin{array}{llll}42 & 08 & 17.3\end{array}$ 420801.5 420757.7 $\begin{array}{llll}42 & 07 & 54.3\end{array}$

420754.3

420753.4

420745.1

\section{7}

$\begin{array}{lll}88 & 58 & 43.0\end{array}$

$\begin{array}{llll}88 & 57 & 50.7\end{array}$

$\begin{array}{lllll}88 & 57 & 33.3\end{array}$

885621.7

$\begin{array}{llll}88 & 56 & 22.0\end{array}$

885511.1

$\begin{array}{llll}88 & 54 & 00.9\end{array}$

885236.6

$\begin{array}{lll}88 & 52 & 32.9\end{array}$

885210.9

$\begin{array}{llll}88 & 51 & 47.5\end{array}$

885103.0

$\begin{array}{lll}88 & 5030.1\end{array}$

$\begin{array}{llll}88 & 49 & 19.5\end{array}$

$\begin{array}{lll}88 & 48 & 45.5\end{array}$

$\begin{array}{llll}88 & 48 & 10.5\end{array}$

$\begin{array}{llll}88 & 47 & 52.8\end{array}$

$\begin{array}{llll}88 & 46 & 59.9\end{array}$

$\begin{array}{llll}88 & 46 & 07.8\end{array}$
5,256

3,940

1,310

5,390

2,650

5,340

5,291

6,350

488

2,771

2,927

0

2,660
5,558

5,558
2,590

2,660

1,340

,

South along highways near east border of quadrangle.

Crossroads.

West quarter corner of sec. 15 , $T$ road west

East-west section line (fence).

Kingston, 0.25 mile north of, center of iron bridge over creek, 780 feet north of Chicago, Milwaukee \& St. Paul Ry. tracks..........

Kingston, Main Street, intersection of .

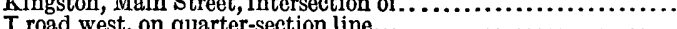

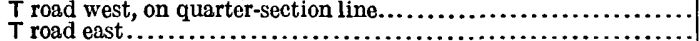

T. 42 N., R. 4 E., secs. 27 and 34 , east-west section-line fence.

Tps. 41 and 42 N., $R .4$ E., line between, T road east..............

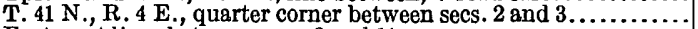

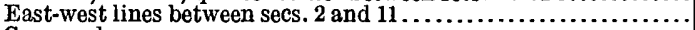

Crossroads.

\begin{tabular}{|c|c|}
\hline $\begin{array}{lll}42 & 07 & 37.2 \\
42 & 07 & 02.2 \\
42 & 06 & 36.2\end{array}$ & $\begin{array}{lll}88 & 45 & 32.7 \\
88 & 45 & 32.5 \\
88 & 45 & 32.2\end{array}$ \\
\hline $\begin{array}{lll}42 & 06 & 10.6 \\
42 & 06 & 00.2 \\
42 & 05 & 17.8 \\
42 & 05 & 04.7 \\
42 & 04 & 51.7 \\
42 & 03 & 59.3 \\
42 & 03 & 36.9 \\
42 & 03 & 10.4 \\
42 & 02 & 44.1\end{array}$ & $\begin{array}{lll}88 & 45 & 31.9 \\
88 & 45 & 31.8 \\
88 & 45 & 31.1 \\
88 & 45 & 30.9 \\
88 & 45 & 30.7 \\
88 & 45 & 29.9 \\
88 & 45 & 15.8 \\
88 & 45 & 12.0 \\
88 & 45 & 02.4\end{array}$ \\
\hline
\end{tabular}

Magnetic declination for center of quadrangle, $3.6^{\circ} \mathrm{E}$.; for east border of quadrangle, $3.5^{\circ} \mathrm{E}$.

North along highways near east border of quadrangle. (By F. W. Crisp in 1917.)

\begin{tabular}{|c|c|c|c|}
\hline 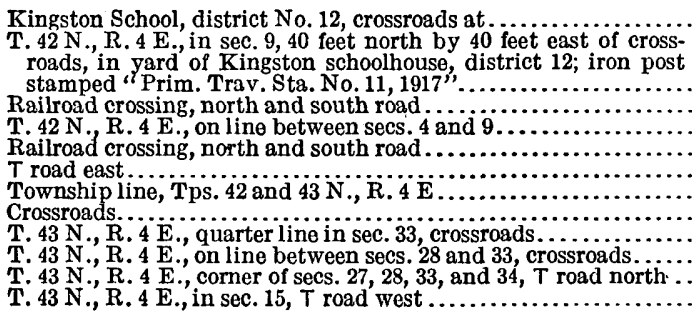 & $\begin{array}{lll}42 & 07 & 45.1 \\
42 & 07 & 45.4 \\
42 & 08 & 01.9 \\
42 & 08 & 20.6 \\
42 & 08 & 46.1 \\
42 & 08 & 46.6 \\
42 & 09 & 13.2 \\
42 & 09 & 26.6 \\
42 & 09 & 39.5 \\
42 & 10 & 05.3 \\
42 & 10 & 05.4 \\
42 & 12 & 03.1\end{array}$ & $\begin{array}{llll}88 & 46 & 07 & 3 \\
88 & 46 & 07.9 \\
88 & 46 & 08.0 \\
88 & 46 & 07.9 \\
88 & 46 & 07.9 \\
88 & 46 & 07.7 \\
88 & 46 & 07.5 \\
88 & 46 & 08.2 \\
88 & 46 & 31.3 \\
88 & 45 & 49.4 \\
88 & 45 & 06.0\end{array}$ & $\begin{array}{r}44 \\
1,674 \\
1,890 \\
2.580 \\
0.55 \\
2,685 \\
1,360 \\
1,301 \\
3,140 \\
3,150\end{array}$ \\
\hline
\end{tabular}

Magnetic declination along north half of east border of quadrangle, $3.3^{\circ} \mathrm{E}$. 


\section{MOUND CITY QUADRANGLE.}

NoTe. $-\Lambda$ adjustment of approximately $3.5^{\prime \prime}$ in latitudo and $1.5^{\prime \prime}$ in longitude has been applied between the east and west borders of this quadrangle. Positions along the Mobile \& Ohio $R$. R. between Eliza triangulation station, Cairo, and Cache, do not contain this adjustment.

East along highways near north border of quadrangle. (By J. H. Wilson in 1916.)

\begin{tabular}{|c|c|c|c|}
\hline Station. & Latitude. & L.ongitude. & $\begin{array}{l}\text { Distance } \\
\text { between } \\
\text { stations. }\end{array}$ \\
\hline 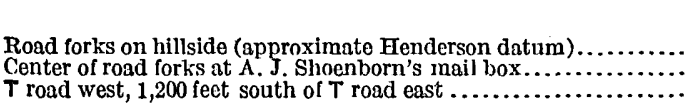 & $\begin{array}{lll}\circ & \prime & \prime \prime \\
37 & 14 & 26.8 \\
37 & 14 & 10.9 \\
37 & 14 & 23.0\end{array}$ & $\begin{array}{lll}\circ & \prime & \prime \prime \\
89 & 04 & 52.8 \\
89 & 04 & 1.8 .6 \\
89 & 02 & 40.1\end{array}$ & $\begin{array}{r}\text { Feet. } \\
\text { 3. } \\
3,200 \\
8,049\end{array}$ \\
\hline
\end{tabular}

Southwest along Big Four Route between Grand Chain and Cairo.

Grand Chain, at fence corner 18 feet north of stock pen, on the west side of track, 180 leet north of depot; iron post stamped "Prim. Trav. Sta. No. 15, 1915 Ill. Elev. 405"

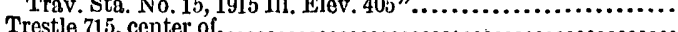

Railroad crossing at north and south road on line between $\mathrm{Rs.} 1$ and $2 \mathrm{E} ., 360$ feet south of railroad crossing is $T$ road east at west corner of secs. 6 and 7, T. 15 S., IR. 2 E

MEAN OF HENDERSON AND NORTH AMERICAN DATUM,

Levings, depot and post office, opposite front door of, center of

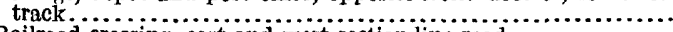

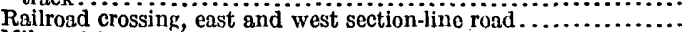
Milepost 244, center of tracit.

Olmstead, railiroad crossing just north of depot, main track.........

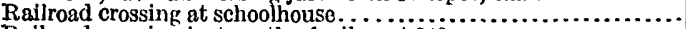

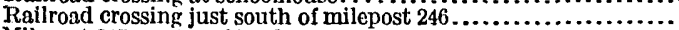
Milepost 247, center of track

Railroad crossing, probable line between secs. 4 and 9 , $\mathrm{T}$. $16 \mathrm{~s}$.,

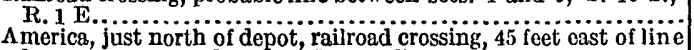

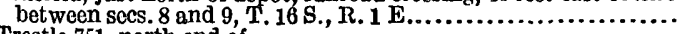
Trestle 751, north end of.

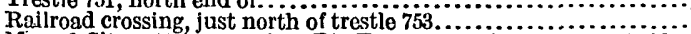

Mound City, 100 feet north of Big Four Route depot, on west side of street leading northwest from depot, on east side of cinder sidewalk, at foot of second telephone pole from depot (U. S. C. E. b. m. No. 64)

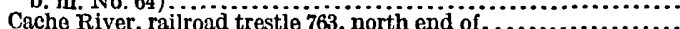

Point of switch

Cairo junction of 3 . directly under shore span of Ilinois Central $R$. $R$. bridge over Ohio River, 25 feet north and 20 feet west of; iron post stamped "Prim. Trav. Sta. No. 8, 1916, n1."

Cairo, Sycamore Street, center track of

Cairo Junction, Illinois Central R. R. (third track from east) directly opposite signal-tower building at junction of Mobilo \& Ohio and Illinois Centrai railroads.
371513.5

371435.8

371346.7

371304.1

371239.0

$37,1132.2$

371056.2

$\begin{array}{lll}37 & 10 & 23.3\end{array}$

$\begin{array}{lll}37 & 10 & 00.7\end{array}$

370917.1

370838.8

$\begin{array}{lll}37 & 08 & 01.1\end{array}$

$\begin{array}{lll}37 & 07 & 08.9\end{array}$

$\begin{array}{lll}37 & 06 & 18.3\end{array}$

$\begin{array}{lll}37 & 05 & 04.4\end{array}$

$\begin{array}{lll}37 & 01 & 12.8\end{array}$

$\begin{array}{lll}37 & 03 & 0 \% .7\end{array}$

370120.4

$37,0118.5$

370220.5
890111.0

890155.0

5,192

890240.5

6,181

$\begin{array}{lll}89 & 03 & 33.2\end{array}$

$\begin{array}{llll}89 & 03 & 47.4\end{array}$

890450.2

$\begin{array}{llll}89 & 05 & 18.0\end{array}$

890543.0

890558.9

$\begin{array}{lll}89 & 06 & 29.7\end{array}$

890656.8

890723.3

$\begin{array}{llll}89 & 08 & 01.6\end{array}$

890836.6

0,045

2,763

8,438

4,260

3,880

2,600

5,045

4,435

4,350

6,285

5,654

890943.1

$\begin{array}{llll}89 & 10 & 22.2\end{array}$

891050.0

9,192

6,087

6,945

\begin{tabular}{lll|l}
89 & 1051.4 & 1,827
\end{tabular}

$891108.2 \quad 1,370$

891130.0

Along Mobile \& Ohio R. R. from Cairo to Beech Ridge.

\section{NORTH AMERICAN DATUM,}

Eliza Point, 50 feet downstream from second or low switchstand, in line with upstream side of cross levee above saw mill and with center of track aloove switches; Iron pipe, 8 inches above surface of ground, outside of track of Mobile \& Ohio R. R. embankment

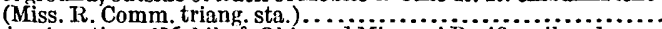
Cairo, junction of Mobile \& Ohio and Missouri Pacific railroads..... Point of switch at north end of $Y$.

T.17 S., R. 1. W., line between secs. 10 and 15 , center of main track, in line with east and west road.

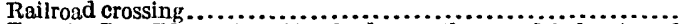

T. $17 \mathrm{~S} ., \mathrm{R} .1 \mathrm{~W}$, center of track, due south of road forks at road north, probably on line between secs. 9 and 10 .

T. $17 \mathrm{~S}$., R. $1 \mathrm{~W}$., center of track at line between secs. 4 and $\ddot{9}$, in line with east and west road, just north of milepost $146 \ldots \ldots \ldots$

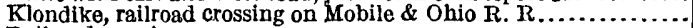

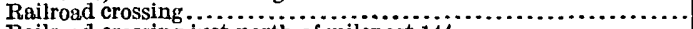
Railroad crossing just north of milepost $144 \ldots \ldots \ldots \ldots \ldots \ldots \ldots \ldots$
370031.43

370122.8 370216.6

370226.9 370233.3 $\begin{array}{lll}37 & 02 & 42.8\end{array}$

$\begin{array}{ll}370319.8 & 0\end{array}$ $\begin{array}{lll}37 & 03 & 38.8\end{array}$

370401.4 $\begin{array}{lll}37 & 04 & 39.3\end{array}$
891138.61

891127.8

$89 \begin{array}{lll}12 & 07.2\end{array}$

5,268

$891234.9 \quad 1,080$

$\begin{array}{lll}891248.8 & 1,485\end{array}$

$891322.5 \quad 4,630$

891346.3

891415.7

891443.9

Magnetic declination near north border of quadrangle, $4^{\circ} 30^{\prime} \mathrm{E}$. 
OREGON QUADRANGLE.

West along highways near north border of quadrangle. (By F. W. Crisp in 1917.)

\begin{tabular}{|c|c|c|c|}
\hline Station. & Latitude. & Longitude. & $\begin{array}{l}\text { Distance } \\
\text { between } \\
\text { stations. }\end{array}$ \\
\hline 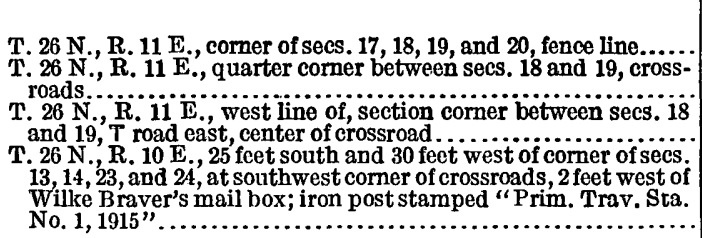 & $\begin{array}{ccc}\circ & \prime \prime \\
42 & 14 & 49.2 \\
42 & 14 & 48.8 \\
42 & 14 & 48.5 \\
42 & 14 & 47.4\end{array}$ & $\begin{array}{cccc}\circ & \prime \prime \\
89 & 15 & 37.0 \\
89 & 16 & 12.2 \\
89 & 16 & 48.7 \\
89 & 17 & 59.0\end{array}$ & $\begin{array}{r}\text { Feet. } \\
\ldots \ldots \ldots \\
2,645 \\
2,745 \\
\\
5,290\end{array}$ \\
\hline
\end{tabular}

South along highways near east border of quadrangle. (By F. W. Crisp in 1917.)

T. 26 N., R. 11 E., west corner of secs. 18, 19, T road east.......

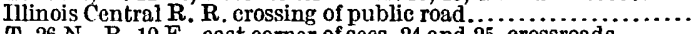
T. 26 N., R. 10 E., east corner of secs. 24 and 25 , crossroads.

i. $26 \mathrm{~N}$,, $\mathbf{R}$. $10 \mathrm{E}$., east corner of secs. 25 and 36 , T road west.... Township corner and south boundary of Winnebago County, $T$ road north.

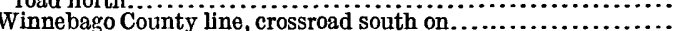

Road elbow south and west on county line.

T road most.

T. 25 N., R. 11 F., line between secs. 7 and 18 , crossroads, 2,690 feet

north of bridge over creek

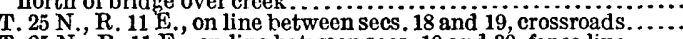

T. 25 N., R. 11 E., on lino between secs. 19 and 30 , fence line.....

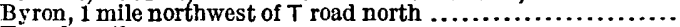

Byron, 0.5 mile west of rain street crossing, on west road bound-

Byron, 0.5 mile west of Main Street crossing, on west road bound-
ary (at city limit), 20 feet south by 25 feet west of cross streets; iron post stamped " Prim. Trav. Sta. No. 5, 1917".............

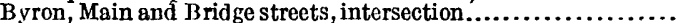

Byron bridge, 500 feet south of sonth end, crossroads.............

T. 24 N., R. 11 E., corner of secs. $5,6,7$, and 8 , T road south, at schoolhouse.

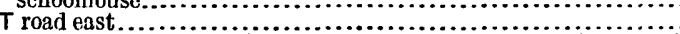

T. 24 N., Rs. 10 and 11 E. 90 feet south by 30 feet west of corner of

secs. 12 and 13 , also 50 feet south by 30 feet west of corner of secs.

7 and 18, at T' road east; iron post stamped "Prim. Trav. Sta.

T. 24 N., R. 11 F., corner of secs. 13 and 24, T road west

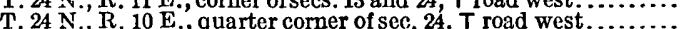

T $24 \mathrm{~N}, \mathrm{R} 11 \mathrm{E}$ quarter corner of sec 19 fence line

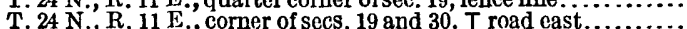

T. 24 N., R. 10 E., quarter corner on east side of sec. 25 , on range

line, if feet north by 20 feet east of $T$ road west; iron post

stamped "Prim. Trav. Sta. No.7, 1917. El. 862".

T. 24 N., R. 11 E., on township line between secs. 31 and $36,{ }^{\top}$ road

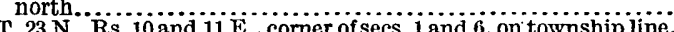

T. 23 N., Rs. 10 and $11 \mathrm{~F}$, corner of secs. 1 and 6 , on township line,

at angle in road...........................................

Crossroads.

T. 23 N., Rs. 10 and $11 \mathrm{E}$. , on line between secs. 1 and $6 \ldots \ldots \ldots \ldots$ No. 6, 1917. E1. 864" .

\begin{tabular}{|c|c|c|}
\hline $\begin{array}{lll}42 & 14 & 48.5 \\
42 & 14 & 17.0 \\
42 & 13 & 56.0 \\
42 & 13 & 03.7\end{array}$ & $\begin{array}{lll}89 & 16 & 48.7 \\
89 & 16 & 47.9 \\
89 & 16 & 47.8 \\
89 & 16 & 47.0\end{array}$ & $\begin{array}{l}3,190 \\
2,130 \\
5,290\end{array}$ \\
\hline $\begin{array}{lll}42 & 12 & 11.7 \\
42 & 12 & 12.0 \\
42 & 11 & 46.3 \\
42 & 11 & 30.4\end{array}$ & $\begin{array}{lll}89 & 16 & 45.6 \\
89 & 16 & 27.0 \\
89 & 16 & 26.0 \\
89 & 16 & 25.7\end{array}$ & $\begin{array}{l}5,260 \\
1,400 \\
2,601 \\
1,606\end{array}$ \\
\hline $\begin{array}{lll}42 & 10 & 28.2 \\
42 & 09 & 35.6 \\
42 & 08 & 43.6 \\
42 & 08 & 17.3 \\
42 & 08 & 17.4\end{array}$ & $\begin{array}{lll}89 & 16 & 25.6 \\
89 & 16 & 25.2 \\
89 & 16 & 24.6 \\
89 & 16 & 24.0 \\
89 & 15 & 55.1\end{array}$ & $\begin{array}{l}6,300 \\
5,330 \\
5,260 \\
2,660 \\
2,180\end{array}$ \\
\hline $\begin{array}{lll}42 & 07 & 35.5 \\
42 & 07 & 36.6 \\
42 & 07 & 12.9\end{array}$ & $\begin{array}{lll}89 & 15 & 48.8 \\
89 & 15 & 20.3 \\
89 & 15 & 14.2\end{array}$ & $\begin{array}{l}4,259 \\
2,151 \\
2,436\end{array}$ \\
\hline $\begin{array}{lll}42 & 06 & 08.5 \\
42 & 05 & 27.4\end{array}$ & $\begin{array}{lll}99 & 15 & 29.5 \\
89 & 15 & 45.1\end{array}$ & $\begin{array}{l}6,52 \\
4,32\end{array}$ \\
\hline $\begin{array}{lll}42 & 05 & 14.8 \\
42 & 04 & 23.4 \\
42 & 03 & 57.2 \\
42 & 03 & 56.6 \\
42 & 03 & 31.0\end{array}$ & $\begin{array}{lll}89 & 16 & 38.0 \\
89 & 16 & 36.6 \\
89 & 16 & 36.2 \\
89 & 16 & 36.2 \\
89 & 16 & 35.5\end{array}$ & $\begin{array}{r}4,193 \\
5,199 \\
2,650 \\
65 \\
2,595\end{array}$ \\
\hline 420305.3 & 891634.7 & 2,600 \\
\hline $4201 \quad 46.9$ & 891633.3 & 7,941 \\
\hline $\begin{array}{lll}42 & 01 & 46.9 \\
42 & 01 & 18.5 \\
42 & 00 & 39.5 \\
42 & 00 & 29.8\end{array}$ & $\begin{array}{lll}89 & 16 & 41.2 \\
89 & 16 & 42.4 \\
89 & 17 & 01.3 \\
89 & 16 & 44.4\end{array}$ & $\begin{array}{r}600 \\
2,880 \\
4,192 \\
1,611\end{array}$ \\
\hline
\end{tabular}

Magnetic declination along east bord er of quadrangle, $4.0^{\circ} \mathrm{E}$.

ROCKFORD QUADRANGLE.

West along highways through center of quadrangle. (By F. W. Crisp in 1917.)

\begin{tabular}{|c|c|c|c|}
\hline Station. & Latitude. & Isongitude. & $\begin{array}{l}\text { Distance } \\
\text { between } \\
\text { stations. }\end{array}$ \\
\hline 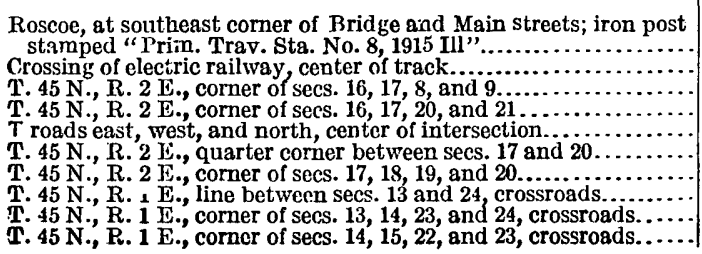 & $\begin{array}{lll}\circ & \prime & \prime \prime \\
42 & 24 & 47.6 \\
42 & 23 & 31.9 \\
42 & 23 & 02.8 \\
42 & 22 & 11.1 \\
42 & 21 & 57.3 \\
42 & 22 & 09.9 \\
42 & 22 & 09.4 \\
42 & 22 & 07.4 \\
42 & 22 & 07.0 \\
42 & 22 & 06.5\end{array}$ & $\begin{array}{llll} & \prime & \prime \prime \\
89 & 00 & 32.8 \\
89 & 00 & 58.0 \\
89 & 01 & 10 & 1 \\
89 & 01 & 09.0 \\
89 & 01 & 08.8 \\
89 & 01 & 44.4 \\
89 & 02 & 19.8 \\
89 & 03 & 56.0 \\
89 & 04 & 40.6 \\
89 & 05 & 48.8\end{array}$ & $\begin{array}{r}\text { Feet. } \\
\ldots ., 900 \\
3,087 \\
5,240 \\
1,390 \\
2,962 \\
2,660 \\
7,222 \\
3,350 \\
5,120\end{array}$ \\
\hline
\end{tabular}


ROCKFORD QUADRANGLE-Continued.

West along highways through center of quadrangle-Cortinued.

\begin{tabular}{|c|c|c|c|}
\hline Station. & Latitude. & Iongitude. & $\begin{array}{l}\text { Distanco } \\
\text { between } \\
\text { stations. }\end{array}$ \\
\hline 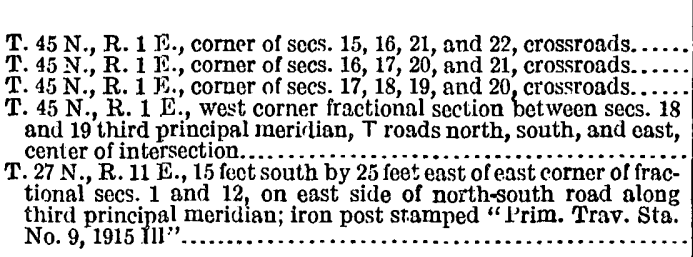 & $\begin{array}{ccc}\circ & , & \prime \prime \\
42 & 22 & 05.9 \\
42 & 22 & 05.5 \\
42 & 22 & 04.9\end{array}$ & $\begin{array}{ccc}\circ & \prime & \prime \prime \\
89 & 06 & 59.9 \\
89 & 08 & 10.6 \\
89 & 69 & 20.6\end{array}$ & $\begin{array}{r}\text { Feet. } \\
5,340 \\
5,310 \\
5,255\end{array}$ \\
\hline
\end{tabular}

South along highweys along third principal meridian.

T. 45 N., R. 1 E., T road at quarter corner of sec. 19 (east cormer on T. $45 \mathrm{~N}$, $\mathrm{R}$. I $\mathrm{N}$, third principal meridian, west side of secs. 19

$\mathbf{T} .27 \mathrm{~N}$., $\mathrm{R}$. $11 \mathrm{i}$., third principal meridian, east side of racT. $45 \mathrm{~N}$., R. 1 F.., third principal meridian, west corner between

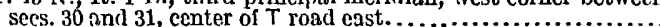
T. 27 N., R. 11 L., third principal meridian, east side corner be-

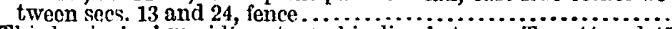
Third principal meridian, township line between $\mathrm{Tp}$. 44 and 15 N., R. 1 l., and west corner between sess. 31 and $6 \ldots \ldots \ldots \ldots \ldots$ T. 27 N., R. 11 F., third principel meridian, quarter corner on east side of sec. 25,30 feet north hy 30 feet west of, center of intersection at $T$ road west; iron post stamped "l'rim. Trav. Sta.

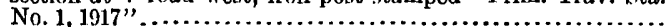

T. 44 N., R. 1 H.., third principal meridian, west section corner T. 44 N., R. 1 I.., third principal meridian, quarter comer on west side of sec. 7 , center of intersectinn at $T$ road east..........

T. 44 N., R. 1 .E., third principal meridian, west section corner

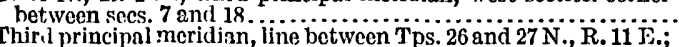
Third principal meridian, line between Tps. 26 and $27 \mathrm{~N}_{\text {. }}, \mathrm{R}_{\text {; }} 11 \mathrm{~F}$.;

T. 44 N., R. $1 \mathrm{l}$., concrete post marked "Third principal merid-

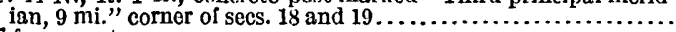

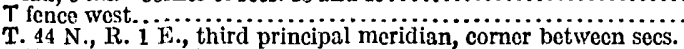
T. $44 N_{\text {, }}$ R. 1 E., third principal meridian, cornor between secs.

T. 44 N., R. 1 F., third principal meridian, corner of secs. 30 and

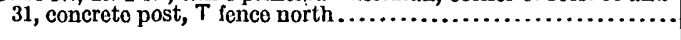

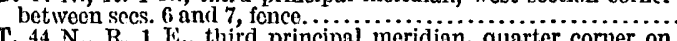

\begin{tabular}{llllll|l}
42 & 21 & 38.1 & 89 & 10 & 26.4 & 1,280 \\
42 & 21 & 11.9 & 89 & 10 & 25.7 & 2,655 \\
42 & 20 & 50.6 & 89 & 10 & 25.9 & 1,345 \\
42 & 20 & 19.7 & 89 & 10 & 25.6 & 3,935 \\
42 & 20 & 06.6 & 89 & 10 & 25.5 & 1,335 \\
42 & 29 & 27.4 & 89 & 10 & 24.8 & 3,970 \\
& & & & & \\
42 & 18 & 49.1 & 89 & 10 & 25.4 & 3,870 \\
42 & 1.5 & 35.6 & 89 & 10 & 24.6 & 1,878 \\
42 & 19 & 09.4 & 89 & 10 & 24.4 & 2,650 \\
42 & 17 & 43.7 & 89 & 10 & 24.2 & 2,600 \\
42 & 17 & 30.1 & 89 & 10 & 24.2 & 1,380 \\
42 & 16 & 59.9 & 89 & 10 & 23.9 & 3,970 \\
42 & 16 & 15.6 & 89 & 10 & 23.5 & 3,573 \\
42 & 15 & 59.2 & 89 & 10 & 23.4 & 1,662 \\
42 & 15 & 07.1 & 89 & 10 & 23.5 & 1,405 \\
\hline
\end{tabular}

Magnetic declination through center of quadrangle, $3^{\circ} \mathrm{E}$; along third principal meridian, $4.4^{\circ} \mathrm{T}$.

West along highways through center of quadrangle. (By F. W. Crisp in 1917.)

\begin{tabular}{|c|c|c|c|}
\hline 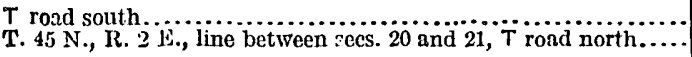 & $\begin{array}{lll}42 & 21 & 57.6 \\
42 & 21 & 57.3\end{array}$ & $\begin{array}{lll}89 & 00 & 51.3 \\
89 & 01 & 08.8\end{array}$ & $\dddot{1,316}$ \\
\hline
\end{tabular}

South along highways near east border of quadrangle. (By F. W. Crisp in 1917.)

\begin{tabular}{|c|c|c|}
\hline $\begin{array}{lll}42 & 21 & 57.3 \\
42 & 21 & 57.6\end{array}$ & $\begin{array}{lll}89 & 01 & 08.8 \\
89 & 00 & 50.9\end{array}$ & $\because, 340$ \\
\hline $\begin{array}{lll}42 & 21 & 18.4 \\
42 & 20 & 25.9 \\
42 & 19 & 49.4 \\
42 & 19 & 33.2 \\
44 & 19 & 32.9 \\
42.19 & 06.6\end{array}$ & $\begin{array}{lll}89 & 00 & 52.0 \\
89 & 01 & 02.8 \\
89 & 01 & 15.3 \\
89 & 01 & 21.3 \\
89 & 01 & 42.1 \\
89 & 01 & 44.2\end{array}$ & $\begin{array}{l}3,969 \\
5,381 \\
3,810 \\
1,700 \\
1,488 \\
2,673\end{array}$ \\
\hline $\begin{array}{lll}42 & 17 & 48.1 \\
42 & 17 & 08.8 \\
42 & 16 & 36.8 \\
42 & 16 & 03.2\end{array}$ & $\begin{array}{llll}89 & 01 & 43.5 \\
89 & 01 & 43.4 \\
89 & 01 & 43.3 \\
89 & 01 & 43.2\end{array}$ & $\begin{array}{l}7,950 \\
3,980 \\
3,240 \\
3,400\end{array}$ \\
\hline $\begin{array}{llll}42 & 15 & 10.7\end{array}$ & $8901 \quad 43.0$ & 5,319 \\
\hline
\end{tabular}


ROCKFORD QUADRANGLE-Continued.

West along highways near south border of quadrangle.

\begin{tabular}{|c|c|c|c|}
\hline Station. & Latitude. & Longitude. & $\begin{array}{l}\text { Distance } \\
\text { between } \\
\text { stations. }\end{array}$ \\
\hline 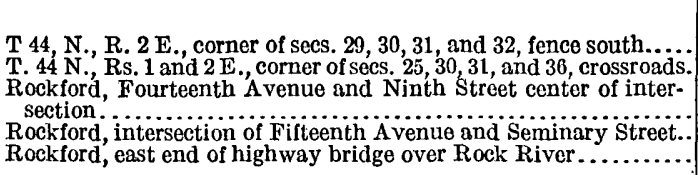 & $\begin{array}{ccc}\circ & \prime & \prime \prime \\
42 & 15 & 10.4 \\
42 & 15 & 10.0 \\
& & \\
42 & 15 & 09.5 \\
42 & 15 & 04.3 \\
42 & 15 & 04.2\end{array}$ & $\begin{array}{ccc}\circ & \prime & \prime \prime \\
89 & 02 & 18.6 \\
89 & 03 & 26.2 \\
& & \\
89 & 04 & 50.3 \\
89 & 05 & 42.7 \\
89 & 06 & 07.6\end{array}$ & $\begin{array}{r}\text { Feet. } \\
2,630 \\
5,090 \\
\\
6,320 \\
3,977 \\
1,870\end{array}$ \\
\hline
\end{tabular}

Magnetic declination for east border of quadrangle, $4^{\circ} \mathrm{E}$.

THEBES QUADRANGLE.

North along Mobile \& Ohio R. R. near east border of quadrangle. (By J. H. Wilson in 1916.

\begin{tabular}{|c|c|c|c|}
\hline Station. . & Latitude. & Longitude. & $\begin{array}{l}\text { Distance } \\
\text { between } \\
\text { stations. }\end{array}$ \\
\hline NORTH AMERICAN DATUM. & $\circ, 11$ & 0,11 & Feet. \\
\hline Beech Ridge, railroad crossing, 1,100 feet south of depot.......... & 370533.0 & 891522.7 & \\
\hline Cache, intersection of Mobile \& Ohio and Illinois Central railroads. & 370606.6 & 891546.9 & 3,915 \\
\hline Cache, 1.5 miles north of, railroad crossing $\ldots \ldots \ldots \ldots \ldots \ldots \ldots \ldots$ & 370710.5 & 891630.4 & 7,363 \\
\hline Railroad crossing, just north of milepost $140 \ldots \ldots \ldots \ldots \ldots$ & 370748.1 & 891629.1 & 3,797 \\
\hline 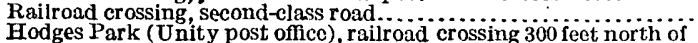 & 370842.4 & 891625.3 & 5,500 \\
\hline depot, line between secs. 1 and 36 , Tps, 15 and 16 S., R. 2 W.... & 370930.7 & 891621.9 & 4,895 \\
\hline $\begin{array}{l}\text { Railroad crossing, } 900 \text { feet south of milepost } 137 \ldots \ldots \ldots \ldots \ldots \\
\text { T. } 15 \text { S., R. } 2 \text { W., line between secs. } 25 \text { and } 36 \text { at second class road }\end{array}$ & $\begin{array}{llll}37 & 10 & 10.0\end{array}$ & $\begin{array}{llll}89 & 16 & 20.9\end{array}$ & 3,993 \\
\hline 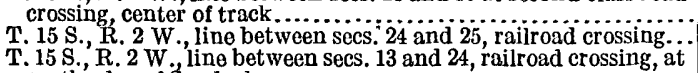 & $\begin{array}{lll}37 & 10 & 23.3 \\
37 & 11 & 16.4\end{array}$ & $\begin{array}{lll}89 & 16 & 29.2 \\
89 & 16 & 20.6\end{array}$ & $\begin{array}{l}1,359 \\
5,408\end{array}$ \\
\hline 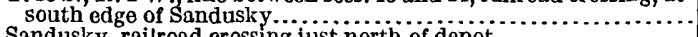 & 371209.4 & 891626.1 & 5,380 \\
\hline 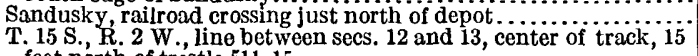 & $\begin{array}{lll}37 & 12 & 30.6\end{array}$ & 891628.8 & 2,155 \\
\hline $\begin{array}{l}\text { feet north of trestle } 511-15 \ldots \ldots \ldots \text { for } \\
\text { Tamms, about } 150 \text { feet south of depot, } 2 \text { feet from high board fence } \\
\text { around hotel yard and } 20 \text { feet northeast of the southwest corner } \\
\text { of this fence; iron post stamped "Prim. Trav. Sta. No.6, 1916, }\end{array}$ & $\begin{array}{lll}37 & 13 & 02.7\end{array}$ & 891629.6 & 3,249 \\
\hline 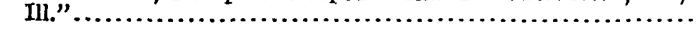 & 371406.4 & 891611.3 & 6,608 \\
\hline
\end{tabular}

Northwest along nlinois Central and Chicago \& Eastern Ilinois railroads from Cache to Gale.

\section{NORTH AMERICAN DATUM.}

Cache 110 feet nor thwest of railroad intersection, at right-angle bend in wagon road, on north side of road, 5 feet west of fence corner; iron post stamped "Prim. Trav. Sta. No. 9, 1916"....... Point of switch

Miller's spur, railroad crossing

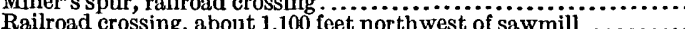

Railroad crossing at second-class road, just north of milepost 138

T. i6 S., R. 2 W., line between secs. 3 and 10, center of track, in

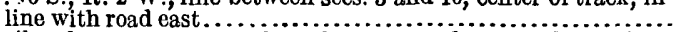

Railroad crossing at north and south road, probably on line

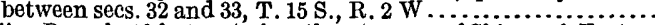

Olive Branch, 10 feet east of northeast corner of Chicago \& Eastern Illinois R. R. station and near edge of platform, on line with south rail of tangent below (northeast of).Olive Branch; iron

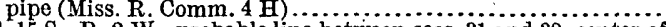

T. 15 S., R. 2 W., probable lino between secs. 31 and 32 , center of

$\mathrm{T}$. $15 \mathrm{~S} ., \mathrm{R} .2 \mathrm{~W}$., probablo line between secs. 31 and 36 , about 600 feet northwest of milepost 371, center of track, in line with.

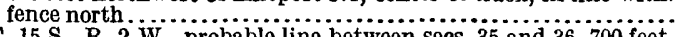

T. $15 \mathrm{~S}$., R. 2 W., probable line between secs. 35 and 36,700 feet west of milepost 372 , railroad crossing..................... Railroad crossing ................................................. Pacific railroads.

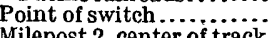

Milepost 2 center

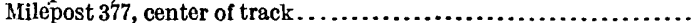

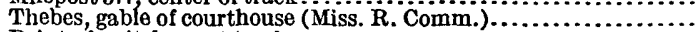

Point of switch, west track.

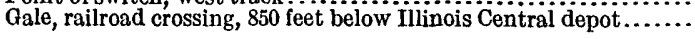

$\begin{array}{llll}37 & 06 & 07.2\end{array}$ $\begin{array}{lll}37 & 06 & 21.1\end{array}$ $\begin{array}{lll}37 & 07 & 29.7\end{array}$ $\begin{array}{llll}37 & 08 & 27.1\end{array}$ 37.0844 .0 $\begin{array}{lll}37 & 09 & 06.7\end{array}$ $\begin{array}{lll}37 & 09 & 51.9\end{array}$

371004.80 370950.1 370947.2 $\begin{array}{lll}37 & 09 & 44.8\end{array}$ $\begin{array}{lll}37 & 09 & 41.8\end{array}$ $\begin{array}{lll}37 & 09 & 43.4\end{array}$ $\begin{array}{lll}37 & 10 & 29.5\end{array}$ $\begin{array}{lll}37 & 11 & 32.7\end{array}$ $\begin{array}{lll}37 & 12 & 20.3\end{array}$ $\begin{array}{lll}37 & 13 & 10.23\end{array}$ $\begin{array}{lll}37 & 14 & 17.9\end{array}$ $\begin{array}{llll}37 & 14 & 43.1\end{array}$ $\begin{array}{llll}89 & 15 & 48.3\end{array}$

891631.2

891751.2

891856.9

891913.4

891935.6

892038.8

3,743

9,496

7,873

2,170

2,910

6,863

892109.39

2, 797

892144.0

3,170

892257.7

5,973

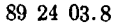

$\begin{array}{lll}89 & 25 & 03.6\end{array}$

5,358

4,855

$\begin{array}{lll}89 & 25 & 19.8\end{array}$

$\begin{array}{lll}89 & 26 & 18.8\end{array}$

$\begin{array}{lll}89 & 27 & 06.0\end{array}$

$\begin{array}{llll}89 & 27 & 28.8\end{array}$

892736.10

892718.5

892658.6
1,318

6,676

7,444

5,157

5,083

6,993

3,009 
THEBES QUADRANGLE-Continued.

Along highways near north border of quadrangle from Thebes to Tamms.

\begin{tabular}{|c|c|c|c|}
\hline Station. & Latitude. & Longitude. & $\begin{array}{l}\text { Disiance } \\
\text { between } \\
\text { stations. }\end{array}$ \\
\hline & 0,11 & 0,11 & Feet. \\
\hline Thebes, center of cross streets at First State Bank $\ldots \ldots \ldots \ldots \ldots \ldots$ & 371312.0 & 892740.2 & \\
\hline Thebes, overhead bridge over Missouri Pacific R. R., west end of. & 371314.9 & 892713.0 & 2,214 \\
\hline Road forks near Beech Grove Church $\ldots \ldots \ldots \ldots \ldots$ & 371322.6 & 892619.2 & 4,423 \\
\hline $\begin{array}{l}\text { Brownville schoolhouse, } 0.5 \text { mile southeast of on east side of road } \\
\text { at road forks, } 5 \text { feet southeast of signboard reading "Diswood } \\
7 \text { miles, Olive Branch } 5 \text { miles;" iron post stamped "Prim. Trav. }\end{array}$ & & & \\
\hline 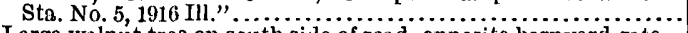 & 3712589 & 892148.2 & 7,739 \\
\hline Large walnut tree on south side of road, opposite barnyard gate. & 371318.6 & 892340.5 & 5,828 \\
\hline 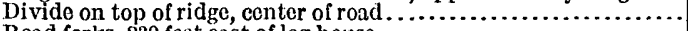 & 371323.1 & 892219.9 & 6,534 \\
\hline $\begin{array}{l}\text { Road forks, } 230 \text { feet east of } \log \text { house } \ldots \ldots \ldots \ldots \ldots \ldots \ldots \ldots \\
\text { Road forks, } 30 \text { feet southeast of walnut tree used as corner post of }\end{array}$ & 371350.2 & $8921 \quad 46.9$ & 3,823 \\
\hline 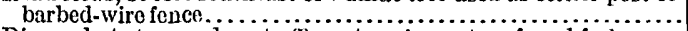 & 371413.5 & 892031.5 & 6,540 \\
\hline Diswood at store and post oflice, stone in center of road forks..... & 371344.2 & 891923.6 & 6,232 \\
\hline 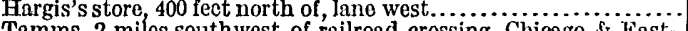 & 37.1317 .2 & 891915.6 & 2,810 \\
\hline $\begin{array}{l}\text { Tamms, } 2 \text { miles southwest of railroad crossing, (hicago \& East- } \\
\text { ern Illinois R. IR., just north of milepost } 13 \ldots \ldots \ldots \ldots \ldots \ldots \ldots \ldots\end{array}$ & 37.1245 .4 & 89.1745 .2 & 7,984 \\
\hline 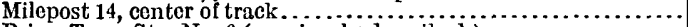 & 371322.4 & 891705.2 & 4,950 \\
\hline Prim. Trav. Sta. No. 6 (previously described ) ..... & 371403.4 & 891611.3 & 6,228 \\
\hline Railroad crossing, 560 feet nor theast of milepost 16. & 371137.5 & 891525.6 & 4,832 \\
\hline
\end{tabular}

Magnetic declination near north border of quadrangle, $5^{\circ} 03^{\prime} \mathrm{E}$.

VERMONT QUADRANGLE.

East along highways near south border of quadrangle. (By J. H. Wilson in 1916.)

\begin{tabular}{|c|c|c|c|}
\hline Station. & Iatitude. & Longitude. & $\begin{array}{l}\text { I)istonce } \\
\text { between } \\
\text { stations. }\end{array}$ \\
\hline 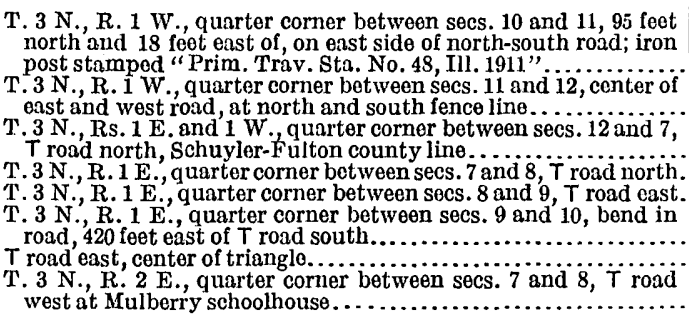 & $\begin{array}{lcc}40 & 15 & 19.4 \\
40 & 15 & 16.6 \\
40 & 15 & 16.0 \\
40 & 15 & 14.5 \\
40 & 15 & 15.0 \\
40 & 15 & 16.1 \\
40 & 15 & 13.3 \\
40 & 15 & 12.6\end{array}$ & $\begin{array}{ccc}\circ & \prime & \prime \prime \\
90 & 29 & 22.2 \\
90 & 28 & 09.6 \\
90 & 27 & 01.5 \\
90 & 25 & 52.9 \\
90 & 24 & 44.6 \\
90 & 23 & 37.5 \\
90 & 19 & 52.6 \\
90 & 19 & 02.2\end{array}$ & $\begin{array}{r}\text { Fect. } \\
\ldots \ldots \ldots \\
5,630 \\
5,284 \\
5,320 \\
\ldots \ldots \ldots \\
5,210 \\
\ldots \ldots \ldots \\
3,905\end{array}$ \\
\hline
\end{tabular}

North along highways near east border of quadrangle.

Tps. 3 and 4 N., R. 2 E., line betwoen secs. 2 and 35 , T road south,

T. 4 N., R. 2 E., quarter corner between sees. 26 and 27 , crossroads.

T. 4 N., R. 2 E., quarter corner between secs. 26 and 27 , crossroads. south road, in line with fence west, large oak tree stands in center

T. 4 N., R. 2 E. cormer of secs. $14,15,22$, and 23 , center of north

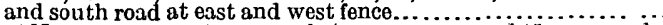
T. 4 N., R. 2 le., quarter corner between secs. 14 ind 15 , crossroads. T. 4 N., $R .2$ E., corner of secs. $10,11,14$, and $15 \ldots \ldots \ldots \ldots \ldots \ldots$ T. 4 N., R. 2 E., quarter corner between sees. 10 and 11 , crossroads. T. 4 N., R. 2 E., corner of sees. $2,3,10$, and $11 \ldots \ldots \ldots \ldots \ldots \ldots \ldots$ T. 4 N., R. 2 E., quarter corner in center of sec. 3 , T road north... Tps. 4 and 5 N., R. 2 E., quarter corner between secs. 3 and 34 .. T. 5 N., R. 2 E., stone corner of secs. $26,27,34$, and 35 , 10 feet north and 3 feet west of, on north side of east and west road at $T$ road south; iron post stamped "Prim. Trav. Sta. No. 12, 1916 Ill". .

Spoon River, near center of triangle at road forks, 100 feet south of

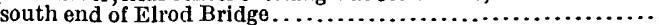

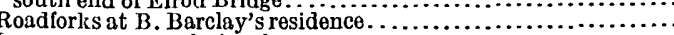

Lane east, center of triangle.

T. 5 N., R. 2 E., corner of secs. $2,3,10$, and $i i$, at bend in road, 10 cot north of large oek tre日...
401631.2

401749.6

401816.2

401909.6

401936.4

402003.1

402029.8

402056.4

402123.5

402150.0

402243.1

402327.7

402505.1

402545.6

402611.5
534

3,823

6,540

232

7,984

,950

, $\mathrm{x} 32$ 
VERMONT QUADRANGLE-Continued.

North along highways near east border of quadranglo-Continued.

\begin{tabular}{|c|c|c|c|}
\hline Station. & Latitude. & Longitude. & $\begin{array}{l}\text { Distance } \\
\text { between } \\
\text { stations. }\end{array}$ \\
\hline 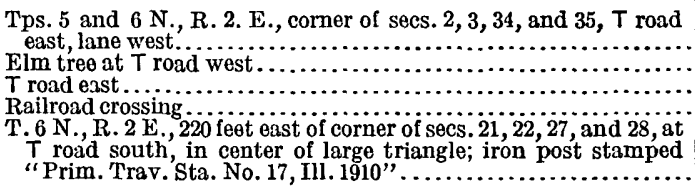 & $\begin{array}{ccc}\circ & \prime & \prime \prime \\
40 & 27 & 05.8 \\
40 & 27 & 25.7 \\
40 & 27 & 45.1 \\
40 & 28 & 23.8 \\
& & \\
40 & 28 & 48.32\end{array}$ & $\begin{array}{ccc}0 & \prime & \prime \prime \\
90 & 15 & 14.2 \\
90 & 15 & 13.3 \\
90 & 16 & 21.3 \\
90 & 16 & 20.5 \\
90 & 16 & 17.21\end{array}$ & $\begin{array}{r}\text { Feet. } \\
5,502 \\
2,010 \\
5,607 \\
3,914\end{array}$ \\
\hline
\end{tabular}

West along highways through center of quadrangle.

T. 5 N., R. 2 E., line between secs. 27 and 28, crossroads at Elrod

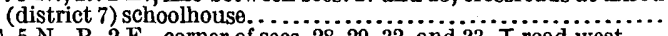
T. 5 N., R. 2 E., corner of secs. $28,29,32$, and 33 , T road west... T. 5 N., R. 2 E., corner of secs. $29,30,31$, and 32 , crossroads at Martin schoolhouse

T. 5 N., R. 2 E., west corner of secs. 30 , and 31 in line with road east, 40 feet south of above corner is T. $5 \mathrm{~N}$., R. 1 E., east corner of secs. 25 and 36 , in line with road west . . ....................

T. 5 N., R. 1 E., T road south, on line between secs. 25 and $26 \ldots . .$.

Road forks at Hawthorne schoollhouse.

T. 5 N., R. 1 E., 20 feet east and several hundred feet south of quarter corner in center of sec. 34, on east side of north and south road at $T$ road west; iron post stamped "Prim. Trav. Sta. No. 13, 1916 IIl."

T. 5 N., R. 1 E., line between secs. 33 and 34

T. 5 N., R. 1 E., south corner of secs. 32 and 33 bend in road, 65 feet south of above corner is T. 4 N., R. 1 E., north corner of secs. 4 and 5 .

Table Grove, cross streets at Christian Church

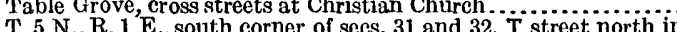
'Table Grove, 45 feet west of above corner is T. 4 N., R. 1 E., north

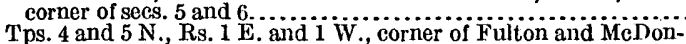
ough county line crossroads at Harris Cemetery, the principal

T. 5 N., R. 1 W., quarter corner south side of sec. 36 , in line with road north, 90 feet east of quarter corner in line with road south.

T. 5 N., R. 1 W., quarter corner between secs. 25 and $36, T$ road

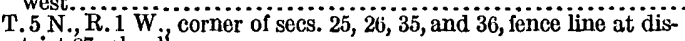
trict 87 schoolhouse.

T. 5 N., R. 1 W., corner of secs. $26,27,34$, and 35,25 feet sonth by 25 feet east of, in southeast corner of $T$ road south; iron post stamped "Prim. Trav. Sta. No. 45, Ill. 1911".

\begin{tabular}{|c|c|c|}
\hline $\begin{array}{lll}40 & 22 & 52.4 \\
40 & 22 & 41.4\end{array}$ & $\begin{array}{lll}90 & 16 & 28.5 \\
90 & 17 & 36.9\end{array}$ & $\cdots, 410$ \\
\hline 402241.2 & $90 \quad 1845.0$ & 5,275 \\
\hline $\begin{array}{lll}40 & 22 & 41.5 \\
40 & 22 & 51.3 \\
40 & 22 & 55.3\end{array}$ & $\begin{array}{llll}90 & 19 & 57.3 \\
90 & 21 & 07.1 \\
90 & 21 & 41.9\end{array}$ & $\begin{array}{l}5,590 \\
5,495 \\
2,727\end{array}$ \\
\hline $\begin{array}{lll}40 & 22 & 08.4 \\
40 & 22 & 09.2\end{array}$ & $\begin{array}{lll}90 & 22 & 51.2 \\
90 & 23 & 25.8\end{array}$ & $\begin{array}{l}7,164 \\
2,680\end{array}$ \\
\hline $\begin{array}{lll}40 & 21 & 51.1 \\
40 & 21 & 51.8\end{array}$ & $\begin{array}{llll}90 & 24 & 35.3 \\
90 & 25 & 26.2\end{array}$ & $\begin{array}{l}5,686 \\
3,940\end{array}$ \\
\hline 402151.9 & 9025.44 .4 & 1,410 \\
\hline 402152.2 & 902653.8 & 5,370 \\
\hline 402152.7 & 902728.4 & 2,680 \\
\hline 402245.5 & 902727.9 & 5,340 \\
\hline $40 \quad 22 \quad 46.4$ & 902802.5 & 2,684 \\
\hline $40 \quad 2247.5$ & 902912.5 & 5,420 \\
\hline
\end{tabular}

Magnetic declination east-west through center of quadrangle, $5^{\circ} 28^{\prime} \mathrm{E}$; f for south border of quadrangle, $5^{\circ} 44^{\prime} \mathrm{E}$.; for east border of quadrangle, $5^{\circ} 12^{\prime} \mathrm{E}$. 


\section{WISCONSIN.}

\section{PRIMARY TRAVERSE.}

Baraboo, Cross Plains, Denzer, Lone Rock, Mazomanie, and White Mound quadrangles.

DANE, IOWA, AND SAUK COUNTIES.

The following geographic positions were determined by primary traverse in 1916 by J. H. Wilson and are based upon primary traverse positions in the Cross Plains quadrangle and upon United States Coast and Geodetic Survey position of Arena Church belfry.

For other positions in Wisconsin seo United States Geological Survey Bulletins 201, 245, 276, 310, 440, 551, and 644; also Part I of the Eighteenth and Twenty-first Annual Reports, United States Coast and Geodetic Survey Report for 1911, as well as unpublished records of the United States Coast and Geodetic Survey, Appendix EEE, Report of Chief of Engineers, United States Army, for 1902, and Mississippi River Commission Report for 1885, 1894, and 1895.

BARABOO QUADRANGLE.

Along highways near southwest corner of quadrangle. (By J. H. Wilson in 1916.)

\begin{tabular}{|c|c|c|c|}
\hline Station. & Latitude. & Longitude. & $\begin{array}{l}\text { Distance } \\
\text { betwoen } \\
\text { stations. }\end{array}$ \\
\hline 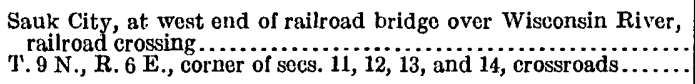 & \begin{tabular}{ccc|}
$\circ$ & $\prime$ & $\prime \prime$ \\
43 & 15 & 56.1 \\
43 & 15 & 50.2
\end{tabular} & $\begin{array}{ccc}\circ & \prime & \prime \prime \\
89 & 43 & 39.7 \\
89 & 44 & 22.3\end{array}$ & $\begin{array}{ll}\text { Fcct. } \\
3,206\end{array}$ \\
\hline
\end{tabular}

Cross plains quadrangie.

(By J. H. Wilson in 1916.)

\begin{tabular}{|c|c|c|c|}
\hline Station. & Latitude. & Longitude. & $\begin{array}{l}\text { Distance } \\
\text { between } \\
\text { stations. }\end{array}$ \\
\hline 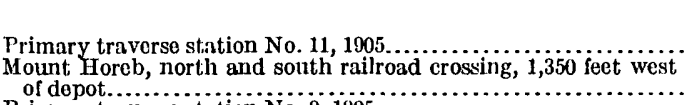 & $\begin{array}{rrr}\circ & 1 & \prime \prime \\
43 & 14 & 58.5 \\
43 & 00 & 21.9\end{array}$ & $\begin{array}{ccc}\circ & , & \prime \prime \\
89 & 43 & 30.2 \\
89 & 44 & 43.6\end{array}$ & $\begin{array}{c}\text { Fcct. } \\
\ldots \ldots . . .\end{array}$ \\
\hline Primary traverse station No. $9,1905 \ldots \ldots \ldots \ldots \ldots \ldots \ldots \ldots \ldots \ldots \ldots \ldots \ldots \ldots \ldots \ldots$ & 430045.5 & $8943 \quad 48.7$ & 4,583 \\
\hline
\end{tabular}


DENZER QUADRANGLE.

West along highways near south border of quadrangle. (By J. H. Wllson in 1916.)

\begin{tabular}{|c|c|c|c|}
\hline Station. & Latitude. & Longitude. & $\begin{array}{l}\text { Distance } \\
\text { between } \\
\text { stations. }\end{array}$ \\
\hline T. 9 N., R. 6 E., line between secs. 14 and 15 , bend in road, 15 & $\circ, \quad \prime \prime$ & $\circ, \prime \prime$ & Feet. \\
\hline 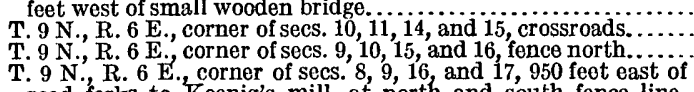 & $\begin{array}{lll}43 & 15 & 06.8 \\
43 & 15 & 49.3 \\
43 & 15 & 50.0\end{array}$ & $\begin{array}{lll}89 & 45 & 34.1 \\
89 & 45 & 33.6 \\
89 & 46 & 44.8\end{array}$ & $\begin{array}{r}\dddot{4}, 300 \\
5,270\end{array}$ \\
\hline 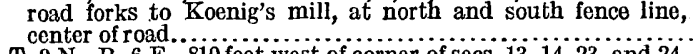 & 431549.8 & 894756.3 & 5,290 \\
\hline 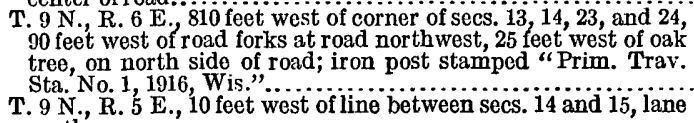 & 431500.0 & 895138.9 & \\
\hline 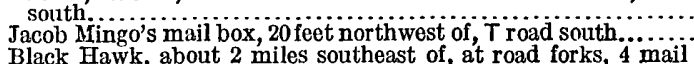 & $\begin{array}{lll}43 & 15 & 02.9 \\
43 & 15 & 18.1\end{array}$ & $\begin{array}{lll}89 & 52 & 40.2 \\
89 & 54 & 08.9\end{array}$ & $\begin{array}{l}4,547 \\
6,743\end{array}$ \\
\hline 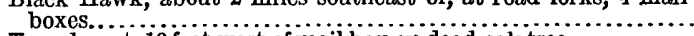 & $\begin{array}{llll}43 & 15 & 25.0\end{array}$ & 895427.1 & 1,521 \\
\hline $\begin{array}{l}\text { T road west, io feet west of mail box on dead oak tree........ } \\
\text { Black Hawk, } 1 \text { mile south of, in line with road north, crossroads. } \\
\text { T. } 9 \text { N., R. } 5 \text { E., line between secs. } 17 \text { and } 18 \text {, just east of old }\end{array}$ & $\begin{array}{llll}43 & 15 & 01.3 \\
43 & 15 & 15.0\end{array}$ & $\begin{array}{lll}89 & 54 & 49.7 \\
89 & 55 & 47.7\end{array}$ & $\begin{array}{l}2,919 \\
4,514\end{array}$ \\
\hline church building, at north and south fence line, center of road. & 431520.3 & 895612.6 & 1,921 \\
\hline 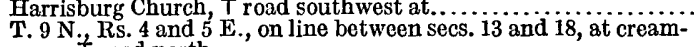 & 431521.8 & 895637.0 & 1,808 \\
\hline 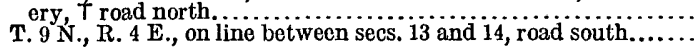 & $\begin{array}{lll}43 & 15 & 24.7 \\
43 & 15 & 36.8\end{array}$ & $\begin{array}{l}895717.6 \\
895830.1\end{array}$ & $\begin{array}{l}3,025 \\
5,500\end{array}$ \\
\hline
\end{tabular}

Magnetic declination for south border of quadrangle, $4^{\circ} 32^{\prime} \mathrm{E}$.

IONE ROCK QUADRANGLE.

South along highways near east border of quadrangle. (By J. H. Wilson in 1916.)

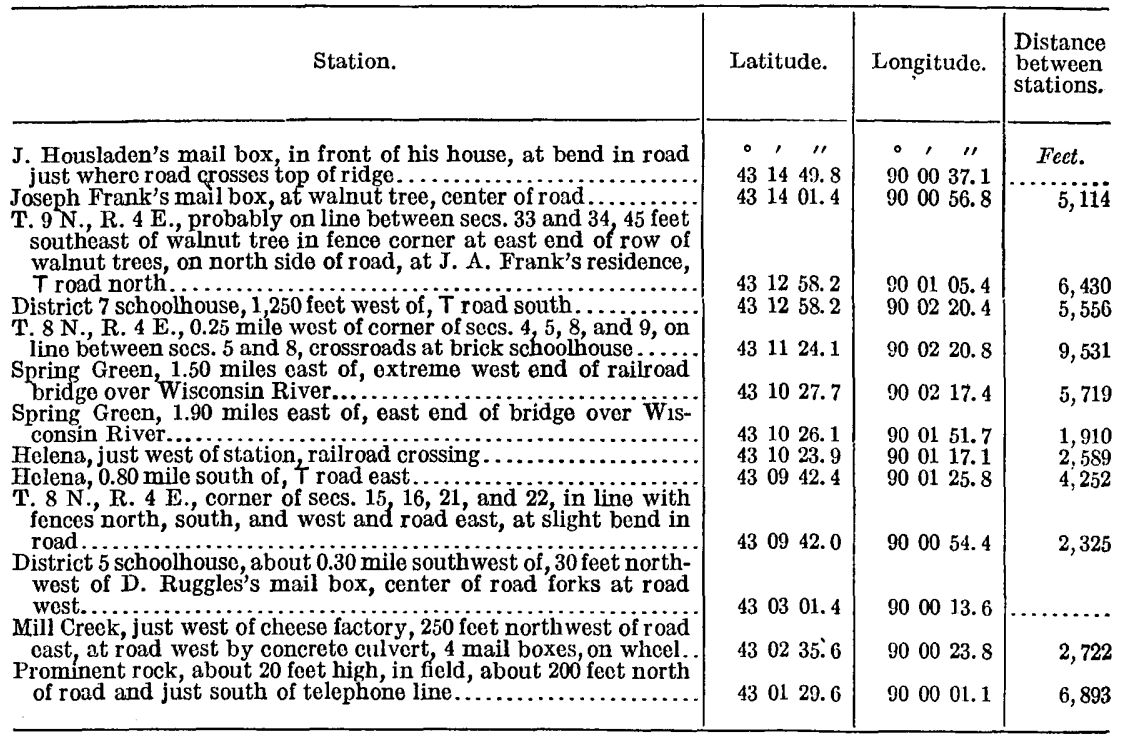

Magnetic declination for east border of quadrangle, $4^{\circ} 47^{\prime} \mathrm{E}$.

MAZOMANIE QUADRANGLE.

South along highways near north border of quadrangle. (By J. H. Wilson in 1916.)

Lane south, center of main road at.

Rich's Corner, 0.25 mile north of Rich's schoolhouse, road forks.

T. 9 N., R. 5 E., approximate corner of secs. 13, 14, 23, and 24 , road
431458.3

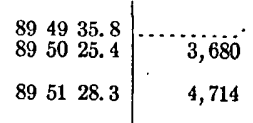


MAZOMANIE QUADRANGLE-Continued.

Along highways near west border of quadrangle.

T. 8 N., R. 4 E., corner of secs. $14,15,22$, and 23

T. 8 N., R. 4 E., quarter corner between secs. 14 and $23, \mathrm{~T}$ road south.

T. 8 N., R. 5 E. quarter corner between secs. 19 and 30 , center of east and west road, in line with road north, about 820 feet east of Bawden schoolhouse.

$T$ road east at Catholic Church

T. $8 \mathrm{~N}$., Rs. 4 and $5 \mathrm{E}$, line between secs. 31 and 36 , center of road nt north and south fence line, 190 feet west of road southeast, at

Road forks, at Mili Creek Church, probably in lino between $\mathrm{T}$ ps. 7 and $8 \mathrm{~N}$

Mill Creek Church, about 600 feet south of, at northwest corner of road forks; Prim. Trav. Sta. No. 4, 1916 Wis.

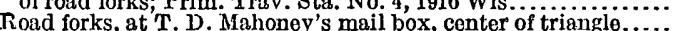

Road forks, about 510 feet west of bridge over Mill Creek, at sign board painted "Spring Green 9 M. Arena $8 \mathrm{M}$."; center of triangle.

T. 7 N., R. 4 E., line between secs. 14 and 23 ; center of rosd at fence

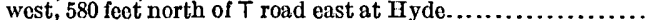

Hyde, about 1 mile south of, at road west, just south of three mail boxes on east side of road.

\begin{tabular}{|c|c|c|}
\hline Latitude. & Longitude. & $\begin{array}{l}\text { Distance } \\
\text { between } \\
\text { stations. }\end{array}$ \\
\hline $\begin{array}{ccc}\circ & \prime & \prime \prime \\
43 & 09 & 42.0\end{array}$ & 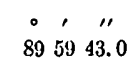 & $\begin{array}{r}F c t . \\
\ldots . . .\end{array}$ \\
\hline $\begin{array}{lll}43 & 09 & 41.9\end{array}$ & 895925.2 & 1,315 \\
\hline $\begin{array}{lll}43 & 08 & 48.9 \\
43 & 07 & 38.8\end{array}$ & $\begin{array}{lll}89 & 56 & 47.6 \\
89 & 57 & 00.3\end{array}$ & $\ddot{7}, 15 \dot{8}$ \\
\hline $\begin{array}{lll}43 & 07 & 19.6\end{array}$ & $\begin{array}{lll}89 & 57 & 20.7\end{array}$ & 2,463 \\
\hline $\begin{array}{lll}43 & 07 & 05.4\end{array}$ & $8957 \quad 50.9$ & 2,654 \\
\hline $\begin{array}{lll}43 & 06 & 59.8 \\
43 & 05 & 43.3\end{array}$ & $\begin{array}{lll}89 & 57.0 \\
89 & 57 & 48.1\end{array}$ & $\begin{array}{r}595 \\
7,752\end{array}$ \\
\hline 430453.9 & $8958 \quad 38.3$ & 6,232 \\
\hline $\begin{array}{lll}43 & 04 & 29.7\end{array}$ & 895851.4 & 2,636 \\
\hline 430358.5 & 895908.8 & 3,410 \\
\hline
\end{tabular}

East along highways through center of quadrangle.

T. 8 N., R. 4 E., corner of secs. $13,14,23$, and 24 , in line with road

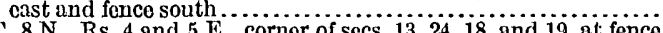
north, center of east and west road.

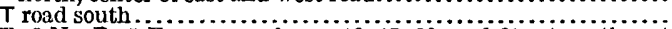
T. 8 N., R. 5 E., corner of sces. $16,17,20$, and 21 , at southwest corner of Arena, $T$ road north.

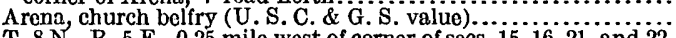
T. 8 N., R. 5 E., 0.25 mile west of corner of secs. 15, 16, 21, and 22, on line between secs. 16 and 21 , crossroads

T. 8 N., R. 5 E., lino between secs. 21 and 28 , center of road at fence east.

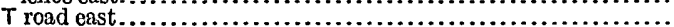

I. 8 N., R. 5 E., lino between secs. 28 and 33 , about 320 feet south of $O$. Nelson's residence, at fence east, center of road........... Tps. 7 and 8 N., R. 5 E., line between secs. 4 and 33,75 feet north of road east, at east and west fence line, center of road .........

T. 7 N., R. 5 E., in northeast part of sec. 4, 180 feet south of road west, on east side of road, at fence corner at road east; iron post stamped "Prim. Trav. Sta. No. 3, 1916 Wis.".

Tps. 7 and 8 N., R. 5 E., line between secs. 3 and 34,10 feet north

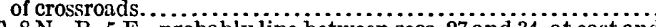

T. 8 N., R. 5 E., probably lino between secs. 27 and 34 , at east and west fence line, center of road

T. 8 N., R. 5 E., 1,450 feet east of crossroads, center of northeast and southwest road, in line with road west through center of

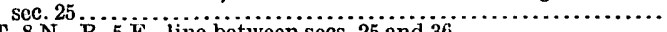

T. 8 N., R. 5 i., line between secs. 25 and 36

T road east 0.5 mile southeast of creamery Blue Mound Creek, center of bridge over, 310 feet east of road fork at Lockwood's residence.

Blue Mound Creek, 1 milo northeast of bridge over, on south side of road, at bend in road at lane north, mail box on fence post

T. 8 N., R. 4 E. probably line between secs. 32 and 33 , about 150 feet northwest of schoolhouse on top of ridge, at fence north,

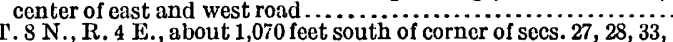
T. 8 N., R. 4 E., about 1,070 feet south of corner of secs. 27, 28, 33, Black Earth, 1.25 miles west of road southwest, center of triangle

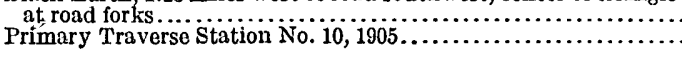

\section{0}

430942.2 430941.8

430941.8 430950.69

430942.1

$\begin{array}{llll}43 & 08 & 49.8\end{array}$ 430823.8

430758.0

430705.6

$\begin{array}{llll}43 & 06 & 43.9\end{array}$

430705.8

430758.2

430825.

$\begin{array}{llll}43 & 07 & 59.3\end{array}$

430737.0

$4307 \quad 15.4$

430733.0

430735.4

430750.2

430829.8 $\begin{array}{lll}43 & 08 & 03.80\end{array}$
895831.4

$\begin{array}{llll}89 & 57 & 19.2\end{array}$ $\begin{array}{llll}89 & 56 & 48.4\end{array}$

$\begin{array}{lll}89 & 55 & 02.3\end{array}$ 895450.28

$\begin{array}{lll}89 & 54 & 08.9\end{array}$

$\begin{array}{lll}89 & 54 & 19.7\end{array}$ 895418.2

$8954 \quad 17.1$

$\begin{array}{lll}89 & 54 & 08.8\end{array}$

895351.8

895348.8

$\begin{array}{lll}89 & 52 & 56.3\end{array}$

895050.5

$\begin{array}{llll}89 & 50 & 46.6\end{array}$

895026.2

894955.1

$\begin{array}{llll}89 & 48 & 49.7\end{array}$

5,355

2,270

7,860

1,264

3,196

5,365

2,631

2,612

5,345

2,538

2,225

6,574

9,733

2,726

3,188

5,170

894756.4

3,964

$89 \quad 46 \quad 45.4$

5,483

$\begin{array}{llll}89 & 46 & 06.1\end{array}$

$894458.20 \quad 4,958$

Magnetic declination for north border of quadrangle, $4^{\circ} 32^{\prime} \mathrm{E}$.; for south border of quadrangle, $4^{\circ} 57^{\prime} \mathrm{E}$.; for west border of quadrangle, $4^{\circ} 47^{\prime} \mathrm{E}$; ; through center of quadrangle, $5^{\circ} 05^{\prime}$. 
MAZOMANIE QUADRANGLE-Continued.

East along highways and Chicago \& North Western Ry. near south border of quadrangle.

Road forks, at road west.

Ridgeway, northeast edge of, at northwest corner of road forks, 80 feet south of railroad crossing and 270 feet south of cemetery; iron post stamped "Prim. Trav. Sta. No. 5, 1916 Wis.".

T. 6 N., R. 4 E., line between secs. 11 and 12 , railroad crossing, 80

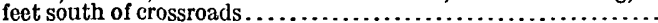

T. 6 N., Rs. 4 and 5 E., quarter corncr between secs. 7 and 12 , center of road, 20 feet west of overhead railroad bridge and 50 feet

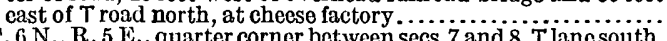
T. 6 N., R. 5 E., quarter corner between secs. 7 and 8 , T lane south, 886 feet east of Junction schoolhouse ......................................

T. 6 N., R. 5 E., quarter corner between secs. 8 and 9 , center of east and west road, at north and south fence line..

Barneveld, 1 mile south west of, railroad crossing signboard roads

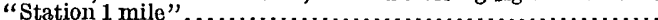

T. 6 N., R. 5 E., quarter corner between secs. 3 and $10, T$ road Barneveld, 1 mile east of, on north side of road at bend in road at Barneveld, 1 mile east of, on north side of road at bend in road at north; iron post stamped "Prim. Trav. Sta. No. 6, 1916 Wis."

Road forks, road south, 123 feet west of District 8 schoolhouse....

T. 6 N., Rs. 5 and $6 \mathrm{E}$, corner of secs. $6,7,1$, and 12 , center of east and west road, 160 fect due south of east side of windmill.........

Blue Mounds, just south of post office, crossing of streets..........

T. 6 N., R. $6 \mathrm{E}$., 120 feet east of north and south line through center of sec. 8, on railroad, directly under overhead road crossing...

T. $6 \mathrm{~N}$, R. $6 \mathrm{E}$, line between secs. 8 and 9 , center of railroad track. Railroad crossing, 375 feet south of schoolhouse...

T. 6 N., R. $6 \mathrm{E}$, north and south road through center of sec. 10 .

T. 6 N., R. 6 E., about 100 feet north of corner of secs. 10, 11, 14 and 15 , center of railroad track, in line with road south; 145 feet above second-class railroad crossing.

\begin{tabular}{|c|c|c|}
\hline Latitude. & Longitude. & $\begin{array}{l}\text { Distance } \\
\text { between } \\
\text { stations. }\end{array}$ \\
\hline $\begin{array}{ccc}\circ & \prime & \prime \prime \\
43 & 01 & 12.9\end{array}$ & $\begin{array}{ccc}\circ & \prime & \prime \prime \\
89 & 59 & 45.0\end{array}$ & $\begin{array}{c}\text { Fcet. } \\
\cdots\end{array}$ \\
\hline $\begin{array}{lll}43 & 00 & 19.8\end{array}$ & $\begin{array}{llll}89 & 59 & 12.6\end{array}$ & 5,887 \\
\hline 430023.6 & S9 5832.3 & 3,015 \\
\hline 430034.3 & 895722.0 & 5,338 \\
\hline $\begin{array}{lll}43 & 00 & 34.4 \\
43 & 00 & 34.2\end{array}$ & $\begin{array}{lll}89 & 56 & 14.6 \\
89 & 55 & 38.7\end{array}$ & $\begin{array}{l}5,015 \\
2,670\end{array}$ \\
\hline $\begin{array}{lll}43 & 00 & 34.2\end{array}$ & 895503.0 & 2,655 \\
\hline $4300 \quad 36.4$ & $8954 \quad 44.2$ & 1,422 \\
\hline $\begin{array}{lll}43 & 01 & 01.0\end{array}$ & $\begin{array}{lll}89 & 53 & 16.8\end{array}$ & 6,954 \\
\hline $\begin{array}{lll}43 & 01 & 01.4 \\
43 & 00 & 55.1\end{array}$ & $\begin{array}{llll}89 & 52 & 49.5 \\
83 & 51 & 27.4\end{array}$ & $\begin{array}{l}2,037 \\
6,131\end{array}$ \\
\hline $\begin{array}{llll}43 & 01 & 01.8 \\
43 & 01 & 02.3\end{array}$ & $\begin{array}{llll}89 & 50 & 18.4 \\
89 & 49 & 55.4\end{array}$ & $\begin{array}{l}5,177 \\
1,709\end{array}$ \\
\hline $\begin{array}{lll}43 & 00 & 52.9 \\
43 & 00 & 51.6 \\
43 & 00 & 25.7 \\
43 & 00 & 21.9\end{array}$ & $\begin{array}{lll}89 & 48 & 33.7 \\
89 & 48 & 00.0 \\
89 & 47 & 06.3 \\
89 & 46 & 12.8\end{array}$ & $\begin{array}{l}6,148 \\
2,510 \\
4,773 \\
3,999\end{array}$ \\
\hline 430011.9 & 894537.6 & 2,806 \\
\hline
\end{tabular}

Magnetic declination near north border of quadrangle, $4^{\circ} 32^{\prime} \mathrm{E}$.; near south border of quadrangle, $4^{\circ} 57^{\prime} \mathrm{E}$; ; near west border of quadrangle, $4^{\circ} 47^{\prime} \mathrm{E}$.; through center of quadrangle, $5^{\circ} 05^{\prime} \mathrm{E}$.

WHITE MOUND QUADRANGLE.

Along highways near southeast corner of quadrangle. (By J. H. Wilson in 1916.)

\begin{tabular}{|c|c|c|c|}
\hline Station. & Latitude. & Longitude. & $\begin{array}{l}\text { Distance } \\
\text { between } \\
\text { stations. }\end{array}$ \\
\hline 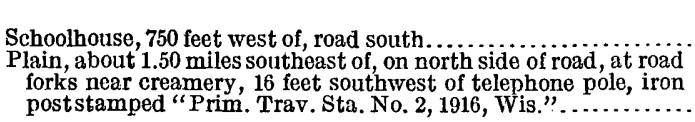 & $\begin{array}{ccc}\circ & \prime & \prime \prime \\
43 & 16 & 00.7 \\
43 & 16 & 17.9\end{array}$ & $\begin{array}{cccc}\circ & \prime & \prime \prime \\
90 & 00 & 00.1 . \\
90 & 01 & 10.3\end{array}$ & $\begin{array}{r}\text { Fect. } \\
\ldots . . . \\
5,481\end{array}$ \\
\hline
\end{tabular}

DELAVAN QUADRANGLE.

(By F. W. Crisp in 1917, extended from work in Illinois.)

\begin{tabular}{|c|c|c|c|}
\hline Station. & Latitude. & Longitude. & $\begin{array}{l}\text { Distance } \\
\text { between } \\
\text { stations. }\end{array}$ \\
\hline T. 1 N., R. 15 E., quarter corner between secs. 29 and 32 , crossroads. & $\begin{array}{ccc}\circ & \prime & \prime \prime \\
42 & 30 & 25.1\end{array}$ & $\begin{array}{ccc}\circ & \prime & \prime \prime \\
88 & 44 & 49.8\end{array}$ & $\begin{array}{r}\text { Feet. } \\
\text {. }\end{array}$ \\
\hline
\end{tabular}

SHOPIERE QUADRANGLE.

(By F. W. Crisp in 1917, extended from work in nlinois.)

\begin{tabular}{|c|c|c|c|}
\hline Station. & Latitude. & Longitude. & $\begin{array}{l}\text { Distance } \\
\text { hetween } \\
\text { stations. }\end{array}$ \\
\hline $\begin{array}{l}\text { T. } 1 \mathrm{~N} ., \text { R. } 14 \mathrm{E} \text {., quarter corner between secs. } 29 \text { and } 32,1,600 \text { feet } \\
\text { west of, north side of east-west road, } 3 \text { feet from plank fence; } \\
\text { iron poststamped "Prim. Trav. Sta. No. 4, 1905" (tie point)... }\end{array}$ & $\begin{array}{ccc}\circ & \prime & \prime \prime \\
42 & 30 & 24.6\end{array}$ & $\begin{array}{ccc}\circ & \prime & \prime \prime \\
88 & 45 & 11.5\end{array}$ & Feet. \\
\hline
\end{tabular}

